\title{
La maison aux esprits : configurations spatiales, pluralité religieuse et syncrétismes dans l'espace domestique afro-cubain
}

The house of the spirits: spatial configurations, religious plurality and syncretisms in the Afro-Cuban domestic space

La casa de los espíritus: configuraciones espaciales, pluralidad religiosa y sincretismos en el espacio domestico afrocubano

\section{Katerina Kerestetzi}

\section{OpenEdition}

\section{Journals}

Édition électronique

URL : https://journals.openedition.org/jsa/15667

DOI : 10.4000/jsa. 15667

ISSN : $1957-7842$

\section{Éditeur}

Société des américanistes

Édition imprimée

Date de publication : 15 juin 2018

Pagination : 75-116

ISSN : 0037-9174

Référence électronique

Katerina Kerestetzi, «La maison aux esprits : configurations spatiales, pluralité religieuse et syncrétismes dans l'espace domestique afro-cubain », Journal de la Société des américanistes [En ligne], 104-1 | 2018, mis en ligne le 15 juin 2018, consulté le 03 septembre 2022. URL : http:// journals.openedition.org/jsa/15667; DOI : https://doi.org/10.4000/jsa.15667 


\title{
La maison aux esprits : configurations spatiales, pluralité religieuse et syncrétismes dans l'espace domestique afro-cubain
}

\author{
Katerina KerestetZI *
}

Cet article propose une réflexion sur le rôle de la spatialité dans la construction de la pluralité religieuse afro-cubaine. La question au cœur de sa problématique est la suivante : comment plusieurs religions peuvent coexister au sein d'un même espace et, surtout, comment l'espace permet à la pluralité religieuse de s'exprimer? À Cuba, en dépit de plus d'un demi-siècle de régime communiste, catholiques, protestants, pratiquants des religions afro-cubaines se rencontrent à tous les niveaux de la société cubaine et dans tous les lieux. Mais ce qui rend le cas cubain également intéressant est que la pluralité religieuse se loge souvent au sein même de la maisonnée, dans laquelle ce sont parfois tous les membres qui pratiquent une religion différente. Ces maisons abritent généralement une vaste gamme d'objets rituels qui peuvent appartenir à des univers forts différents - chaudrons du palo monte recouverts de sang, statues et icônes de saints catholiques, effigies de la santería... Comment l'espace domestique est-il aménagé en espace rituel ? Selon quelles logiques rituelles et réflexives ? Comment est-il partagé, compartimenté afin de répondre aux exigences des différents cultes, souvent antinomiques ? Les objets rituels présents dans une maison sont perçus par les pratiquants comme de véritables incarnations des dieux et des esprits, dotées d'intentionnalité, de personnalité et de préférences. De même qu'il existe des possibilités de symbiose, il existe aussi des agencements contre-productifs qui requièrent une grande inventivité rituelle ou un usage ingénieux de l'espace. En outre, les ménages cubains de spiritualité mixte sont le siège d'une intense ritualité, celle du quotidien mais aussi celle des cérémonies collectives qui rassemblent l'ensemble de la communauté. L'étude de l'inscription topographique de cette ritualité au sein de l'espace domestique révèlera les interactions et les tensions entre les différentes pratiques religieuses, ainsi que les micro-ajustements nécessaires et créatifs qui leur permettent de coexister au sein d'un espace partagé. [Mots-clefs : pluralité religieuse, religions afro-cubaines, spatialité, esthétique, rituel.]

The house of the spirits: spatial configurations, religious plurality and syncretisms in the Afro-Cuban domestic space. This article proposes an examination of the role of spatiality in the construction of Afro-Cuban religious plurality. The question at

*CNRS, Laboratoire d'anthropologie sociale [katerina.kerestetzi@college-de-france.fr]. 
the heart of its problematic is the following: how can different religions coexist within the same space, and, especially, how can space enable the expression of religious plurality? In Cuba, Catholics, Protestants, and practitioners of Afro-Cuban religions meet everywhere and at all levels of society despite over half a century of communist rule. But what makes the Cuban case particularly interesting is that religious plurality is often found within a single household, which can accommodate as many religions as there are family members. Such homes generally house a wide range of ritual objects belonging to different religious worlds, such as blood-covered Palo Monte pots; statues and icons of Catholic saints; and effigies of Santería's deities. How is the domestic space transformed into a ritual one? What are the ritual, cosmological, and reflexive logics enabling such a transformation? How is the household arranged and divided? How are the different religious objects displayed in order to respect the needs and conceptions of each of the various, and often contradictory, religious philosophies? Representing more than just things, Afro-Cuban ritual artifacts are seen by practitioners as true incarnations of gods and spirits, imbued with agency, personality, and preferences. Just as there are opportunities for symbiosis, there are also counterproductive layouts requiring considerable ritual inventiveness and clever use of space. This article will reveal an ecology of ritual objects. Moreover, Cuban households of mixed spirituality are centers of intense rituality. By rituality I am referring to private ritual actions performed on a daily basis but also to the collective ceremonies that bring together the whole religious community. The study of the topographical inscription of this rituality within the domestic space highlights the interactions and tensions between different religious practices as well as the necessary and creative micro-adjustments that allow them to coexist within a shared space. [Key words: religious plurality, Afro-Cuban religions, spatiality, aesthetics, ritual.]

La casa de los espíritus: configuraciones espaciales, pluralidad religiosa y sincretismos en el espacio domestico afrocubano. Este artículo propone una reflexión sobre el papel de la espacialidad en la construcción de la pluralidad religiosa afrocubana. La pregunta en el corazón de su problemática es la siguiente: ¿Cómo varias religiones pueden coexistir en el mismo espacio y, sobre todo, cómo el espacio permite a la pluralidad religiosa de expresarse? En Cuba, a pesar de más de medio siglo de gobierno comunista, los católicos, los protestantes y los practicantes de las religiones afrocubanas se encuentran en todos los niveles de la sociedad y en todo lugar. Pero lo que hace el caso cubano también interesante es que la pluralidad religiosa se encuentra a menudo en la misma casa donde, a veces, cada miembro de la familia pratica una religión diferente. Estas casas abrigan por lo general una amplia gama de objetos rituales que pueden pertenecer a universos muy diferentes: cazuelas de palo monte cubiertas de sangre, estatuas e imágenes de santos católicos, efigies de santería... ¿Cómo el espacio doméstico se convierte en un espacio ritual? De acuerdo con cuales lógicas rituales y reflexivas? ¿Cómo se comparte y se reparte para satisfacer las necesidades y las lógicas de los diversos cultos, a menudo contradictorias? Los objetos rituales presentes en una casa son vistos por los practicantes como verdaderas encarnaciones de dioses y de espíritus, con intencionalidad, personalidad y preferencias. Al igual que hay posibilidades de simbiosis, también existen configuraciones contra-productivas que requieren una gran inventiva y un 
uso ingenioso del espacio. Además, los hogares cubanos de espiritualidad mixta son el lugar de una intensa ritualidad, del cuotidiano, pero también de las ceremonias colectivas que reúnen a toda la comunidad. El estudio de la inscripción topográfica de esta ritualidad en el espacio doméstico revelerá las interacciones y las tensiones entre las diferentes prácticas religiosas y los microajustes necesarios y creativos que les permitan coexistir en un espacio compartido. [Palabras claves: pluralidad religiosa, religiones afrocubanas, espacialidad, estética, ritual.]

Cette recherche propose une réflexion méthodologique sur le rôle de la spatialité dans l'activité religieuse en contexte de pluralisme religieux. La question qu'elle pose est la suivante : comment plusieurs religions peuvent-elles coexister au sein d'un même espace et, surtout, comment l'espace permet-il à la pluralité religieuse de s'exprimer ? À Cuba, en dépit de plus d'un demisiècle de régime communiste, la pratique religieuse est intense et la pluralité de religions s'épanouit sans dissonance particulière. Catholiques, protestants, pratiquants des religions afro-cubaines, de la santería et du culte d'Ifá (toutes deux d'influence yoruba), du palo monte (d'influence bantoue), du spiritisme syncrétique (d'influence européenne-nord-américaine et africaine) se rencontrent partout et à tout niveau de la société cubaine - le foisonnement des religions n'est pas cantonné aux seules grandes villes cosmopolites. Ce qui rend le cas cubain intéressant est que cette pluralité religieuse se rencontre aussi sous le même toit, chacun des membres d'une même famille pouvant pratiquer un culte différent. Cette configuration offre la possibilité d'étudier les interactions quotidiennes entre différentes pratiques religieuses et les micro-ajustements nécessaires qui leur permettent de coexister au sein d'un espace partagé.

Avec la fermeture au début du $\mathrm{Xx}^{\mathrm{e}}$ siècle des cabildos de nación - sortes d'associations d'entraide qui servaient aussi de lieux de culte pour les esclaves africains et leurs descendants ${ }^{1}-$, l'essentiel des pratiques rituelles s'est déplacé dans les espaces domestiques que les pratiquants et certains chercheurs (voir Gónzalez Huguet 1968) désignent aujourd'hui sous le vocable de casas-templos (maisons-temples). Ces maisons sont devenues les véritables sanctuaires des cultes afro-cubains. La coexistence en leur sein d'individus pratiquant plusieurs religions est le produit de nombreux facteurs sociohistoriques. La raison la plus évidente tient à la grande difficulté pour les Cubains d'obtenir une habitation individuelle, conséquence d'une pénurie de logements à l'échelle du pays, mais aussi de procédures administratives particulièrement ardues. Jusqu'en 2011,

1. Dès les débuts de la colonisation et sous l'impulsion de la couronne espagnole, les esclaves africains ont créé ces associations de secours mutuel, qu'on appelle aussi « cabildos » (littér. « corporation » ou « conseil municipal »). On réunissait des fonds pour aider les malades, payait les funérailles, achetait la liberté de certains esclaves, mais aussi on organisait les fêtes religieuses et les rites. C'est leur mauvaise réputation qui a entraîné leur suppression (Ortiz 1986 [1926] ; Brown 2003). 
il n'était par exemple pas possible pour un Cubain d'acheter ou de vendre une maison, mais uniquement de l'échanger. Malgré l'assouplissement de la loi, la situation aujourd'hui reste inchangée car la plupart des Cubains n'ont tout simplement pas les ressources nécessaires pour s'acheter un logement. Par conséquent, les enfants devenus adultes, même en couple, sont souvent obligés de partager la maison avec leurs parents et il n'est pas rare que trois ou quatre générations cohabitent sous le même toit. Les ménages cubains sont très hétérogènes car ils comptent une grande proportion de familles recomposées. Le taux de remariage, très élevé à Cuba, relève à la fois de la simplicité de la procédure de divorce et du peu de prégnance du modèle de la famille nucléaire - ce dernier point étant considéré par certains auteurs comme une conséquence des modes de vie mis en place pendant la période de l'esclavage à Cuba (André 1987 ; Stolcke 1992).

Les maisons cubaines aux spiritualités multiples abritent aussi un nombre impressionnant d'objets rituels, de taille souvent imposante. L'espace domestique peut ainsi être saturé d'objets religieux. Dans une maison-temple, il m'est arrivé de recenser jusqu'à 300 artefacts associés à quatre religions différentes. Les expressions matérielles propres à chaque culte sont variables par leur nombre et leur forme. Un santero peut par exemple implanter dans sa maison plusieurs divinités (orichas) et en décliner la représentation de chacune sur différents supports (récipients, effigies, colliers rituels). Les pratiquants du palo monte donnent corps à leurs morts inquiétants en les intégrant dans d'imposants chaudrons débordant de matière organique. Les spirites rendent présents leurs morts familiaux en tapissant leurs autels domestiques de leurs photographies. Quant à Jésus ou parfois à Bouddha ou à Shiva - chaque figure religieuse populaire peut y avoir sa place -, on les retrouve sous forme d'icônes ou de statuettes qui viennent s'ajouter aux posters des héros de la révolution cubaine, eux-mêmes devenus des symboles de pouvoir utilisés à des fins rituelles...

Cette polyphonie figurative est accompagnée d'une intense ritualité qui permet d'observer à un niveau routinier l'articulation spatiale des différentes pratiques religieuses. Par ritualité, il faut aussi bien comprendre celle du quotidien, des oraisons intimes, de petites offrandes de tabac et de rhum faites aux différentes entités que celles, plus exceptionnelles, de fêtes collectives qui réunissent toute la communauté. C'est à ces occasions, qui densifient les populations humaines et spirituelles de la maisonnée, qu'on assiste à la mise en place des plus spectaculaires compromis spatiaux ; certaines pièces, comme le salon, étant littéralement réaménagées pour s'adapter à l'agenda spirituel de chacun. Cet article s'intéresse à la manière dont l'espace habité est aménagé en espace rituel et aux techniques de compartimentation employées pour respecter les besoins des différents cultes mis en présence.

Il faut préciser que les objets rituels afro-cubains ne sont pas pour les pratiquants de simples représentations, mais bel et bien de véritables incarnations 
de divinités et d'esprits, dotées d'une intentionnalité, d'une capacité d'action autonome, mais aussi d'une personnalité, de sentiments et de besoins. Leur « tempérament » particulier leur confère une capacité plus ou moins grande à se mêler aux autres objets. De même qu'il existe des possibilités de symbiose, il existe aussi des rapprochements dangereux ou contre-productifs qui requièrent une grande inventivité rituelle ou un usage ingénieux de l'espace. S'interroger sur leur positionnement dans la maison revient ainsi à aborder la question d'une « écologie des objets rituels ».

Nous examinerons la manière dont la cosmologie de chaque culte interagit avec la spatialité domestique et la manière dont les entités antagonistes trouvent leur place dans l'écosystème d'une maison-temple. L'objectif est de dégager les principes généraux qui sous-tendent la coexistence des différents objets rituels au sein de l'habitat. Nous mettrons ainsi en rapport la pratique religieuse, la spatialité et les rapports de famille afin de montrer qu'ils sont constitutifs les uns des autres ; les rapports familiaux se muant en composantes rituelles et les « affordances » (Gibson 1977) spatiales en indices cosmologiques. L'examen d'un échantillon de cas concrets d'habitats plurireligieux nous permettra en outre d'identifier certaines modalités du « travail du syncrétisme » (Mary 2000) afro-cubain.

Les exemples qui vont être cités concernent des maisons et des appartements de Cienfuegos et de Palmira, villes situées dans la partie centrale de l'île de Cuba où j'ai mené l'essentiel de cette enquête. Par habitat plurireligieux, je désigne aussi bien les logements partagés par des pratiquants de religions différentes que ceux où un seul et même individu est affilié à de multiples cultes. En effet, un grand nombre de pratiquants sont de manière régulière ou occasionnelle impliqués simultanément dans plusieurs cultes, de sorte que l'on peut être à la fois palero, santero, spirite et catholique (et communiste). La première catégorie d'exemples nous permettra de centrer l'analyse sur les compromis qui permettent de trouver un équilibre entre les intérêts et les divinités des différents membres de la maisonnée, tandis que la seconde permettra de mieux comprendre comment une personne organise son appartenance multiple et exprime, dans son espace, sa manière personnelle de concevoir cette pluralité.

La recherche anthropologique sur les religions afro-cubaines s'est déjà abondamment intéressée à la question de la pluralité religieuse, en insistant sur la complémentarité des différents cultes afro-cubains (López Valdés 1985 ; Argyriadis 1999 ; Dianteil 2000), ou sur la manière dont ces derniers interagissent conceptuellement et rituellement (Palmié 2002 ; Espírito Santo 2015). En même temps, certains auteurs ont fourni de brillantes descriptions d'autels et d'objets de la santería cubaine et de ses parures (Brown 2003 ; Flores-Peña et Evanchuk 1994 ; Mason 1992). Or une approche qui traite à la fois de la spatialité, de la matérialité et de la pluralité religieuse fait encore défaut. Une étude systématique des espaces domestiques cubains manque aussi pour l'instant. 
Dans le champ plus large des études afro-américaines, la problématique spatiale a connu un relatif intérêt, notamment au Brésil. Même s'il n'existe aucun ouvrage à proprement parler dédié à la question de la spatialité, plusieurs auteurs consacrent des chapitres entiers de leurs livres à des descriptions des sanctuaires du candomblé et de l'umbanda par exemple, et à leur topologie. Leur perspective, dans la majorité des cas d'inspiration structuraliste, oppose la rue au lieu rituel, le dehors au dedans, le centre à la périphérie (Giobellina Brumana 1994). Leur objectif est de dégager les dynamiques sociologiques à l'œuvre dans ces cultes. Par exemple, la « subalternité topologique » (la périphérie) est associée à la « subalternité religieuse » (Giobellina Brumana et Gonzales Martínez 1989) ou bien le « dedans » est vu comme un mécanisme d'édification du " secretism », un mécanisme régulateur des relations au sein du candomblé (Johnson 2002). Dans la continuité de ces passionnants travaux, qui vont d'ailleurs au-delà du modèle dichotomique auquel ils souscrivent, il s'agit ici de monter que la valeur heuristique d'une approche spatiale tient au fait qu'elle dépasse le dialectisme qu'implique le va-et-vient entre sujet et matière. L'espace, étant partagé et construit collectivement, devient l'expression objectivée d'une multiplicité de relations, d'idées et de notions. En tant qu'outil méthodologique, il permet aussi d'analyser les processus idiosyncrasiques qui sous-tendent l'édification des pratiques religieuses, tout en les mettant en rapport avec des dynamiques éminemment sociologiques (rapports entre membres, relations de voisinage, représentations sociales). Pour le dire autrement, la spatialité ouvre la voie d'une analyse qui peut à la fois être micro et macroscopique. En remontant le fil des raisons qui ont assigné aux objets rituels leur place dans l'espace domestique, l'ambition est d'objectiver les multiples dimensions du social et de l'appartenance religieuse, qu'elles soient relationnelles, esthétiques, représentationnelles, économiques ou idéologiques.

\section{Esthétiques cosmologiques : les objets rituels afro-cubains}

Puisque cet article traite de la pluralité religieuse sous l'angle de la matérialité et de la spatialité, une brève description des différents objets et styles esthétiques des cultes présents dans une maison-temple s'impose afin de comprendre ce qui est amené à cohabiter sous le même toit.

\section{La santería/Ifá : luxe et mystère}

Commençons par la santería, autrement appelée « Regla Ocha », et le culte d'Ifá qui partagent la même cosmologie et le même univers matériel. Leurs adeptes, respectivement les santeros et les babalaos, utilisent le terme générique de fundamento (littér. «fondement ») pour désigner les artefacts qui incarnent leurs divinités prestigieuses, les orichas. Or cette appellation cache en réalité une grande diversité figurative : soupières en porcelaine, statuettes d'inspiration 


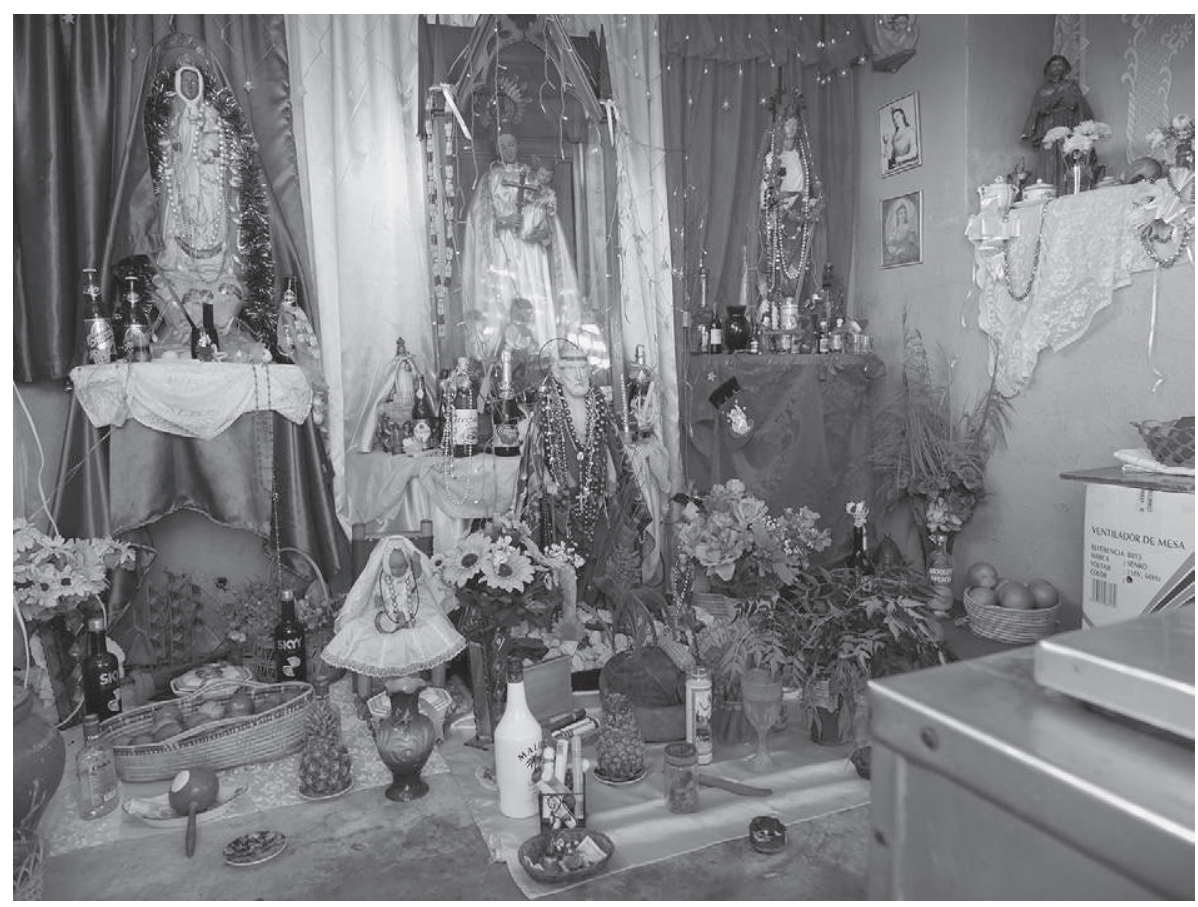

Fig. 1 - Sanctuaire de santería.

africaine, poupées, bibelots, statues de saints catholiques... (Figures 1,2). On y distingue des styles différents, inspirés de sources hétérogènes. On peut parler d'une « esthétique syncrétique » qui emprunte aussi bien aux autres cultes présents à Cuba (catholicisme, palo) qu'à la mode décorative cubaine et même à certaines représentations historiques (l'africanité de la santería, la royauté des colons espagnols, la bourgeoisie ou les indigènes disparus, voir Palmié 2002).

Si les critères de beauté plébiscités par les santeros et les babalaos cubains ne sont pas forcément partagés par tous, l'intention des pratiquants de créer du beau ne fait aucun doute : on trouve du brillant, du flamboyant et du propre, valeurs esthétiques partagées par bien des fidèles à Cuba (Argyriadis 1999). On utilise des matériaux « nobles »-porcelaine, velours, satin, or, argent (ou des imitations), des fleurs et du parfum. Et on utilise beaucoup de couleur. La mythologie même de la santería invite les pratiquants à construire un lieu de culte très coloré, chaque oricha étant associé à une ou plusieurs couleurs. Yemayá, la déesse de la mer, est représentée par le bleu et le blanc ; Obatalá, le dieu de la sagesse qui domine les maisons, se distingue par la blancheur immaculée de ses parures... Le rouge, le vert, le noir, le marron sont également utilisés. Les autels santeros hébergent généralement l'intégralité du panthéon 


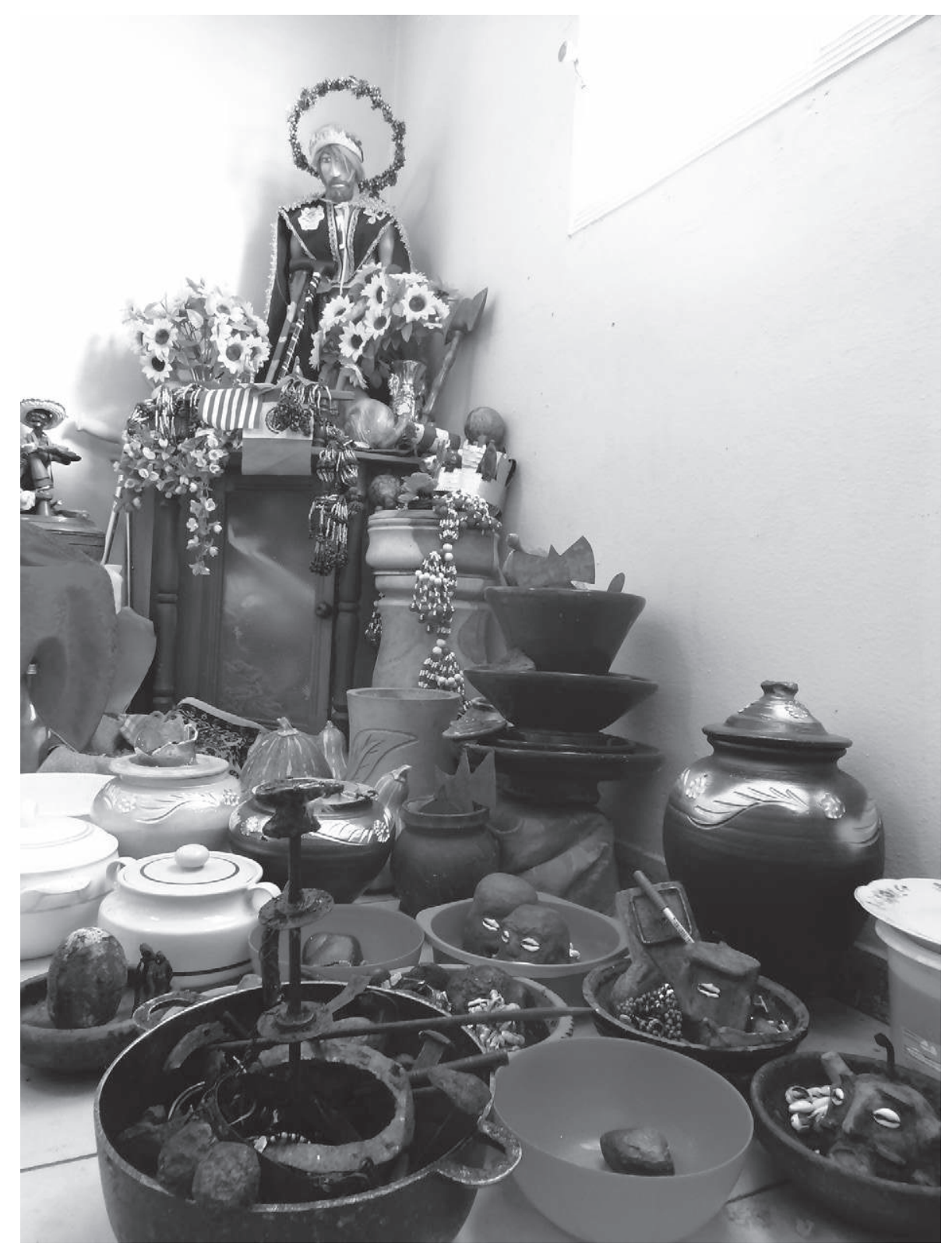

Fig. 2 - L'essentiel des objets de la santería, rassemblés dans un même coin lors d'un rituel pour recevoir le sang des sacrifices et d'autres offrandes « alimentaires ». 


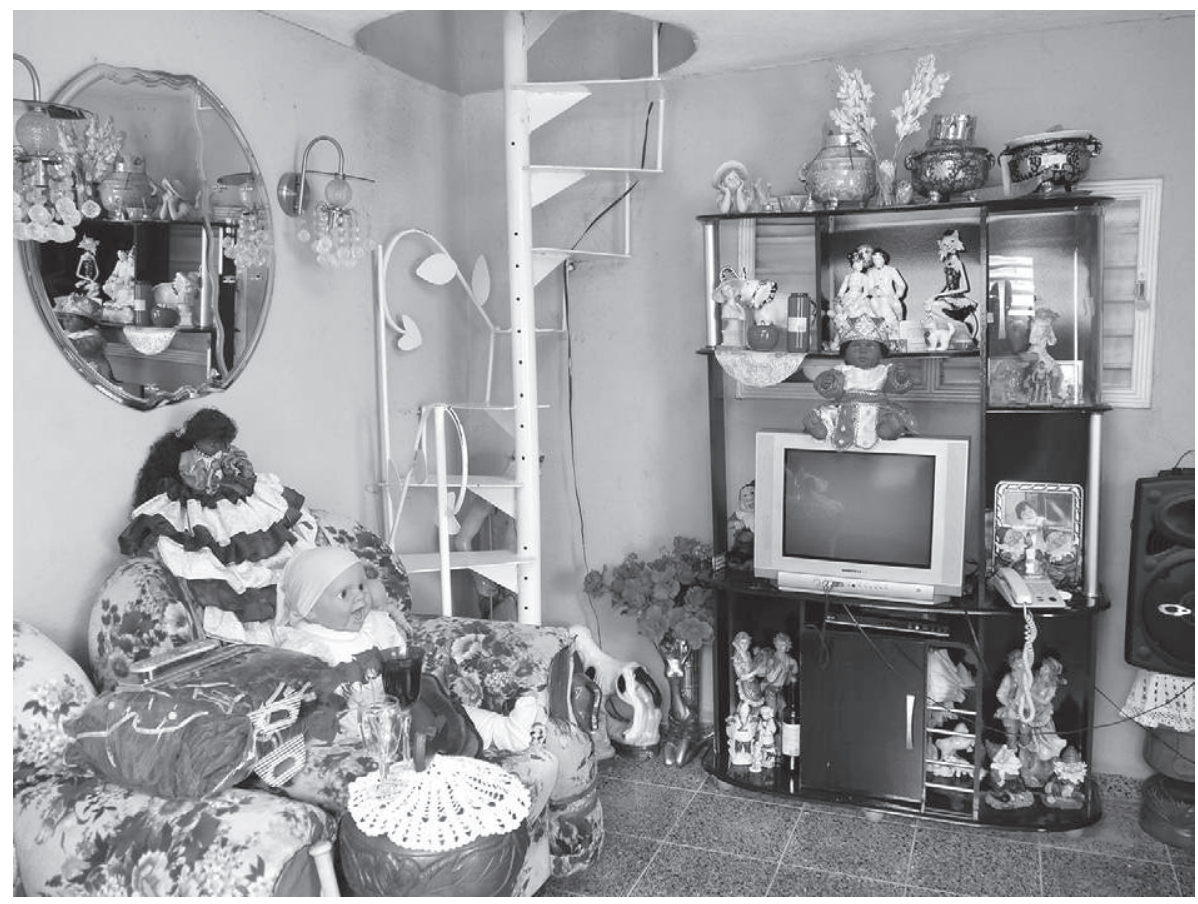

Fig. 3 - Salon d'une maison de spiritualité mixte. Les poupées installées sur le fauteuil et au-dessus de la télévision sont des orichas, des incarnations des divinités de la santería. Les soupières qui sont posées au sommet du meuble de la télévision sont également des orichas. Derrière l'escalier, figure saint Lazare avec ses béquilles et ses chiens. Certains bibelots correspondent aussi à des entités spirituelles ainsi qu'un objet, nommé " pilón », qui sert de siège pour les aspirants santeros lors de leur initiation. Cet objet en bois est posé devant les fauteuils (il est couvert d'une broderie).

oricha, ce qui produit une impressionnante polychromie. Le luxe aussi est suggéré symboliquement dans le culte. En fait, certaines des divinités incarnent paradoxalement à la fois un idéal de la nature et un esprit mondain : Ochún, par exemple, la maîtresse des eaux douces, est aussi l'oricha de la prospérité qui s'épanouit dans la richesse.

Cette catégorie d'artefacts à caractère domestique, voire décoratif, comporte aussi des peluches et des bibelots représentant des animaux, mais aussi des poupées pour enfants (en chiffon, en plastique, en plâtre). Là encore, il s'agit d'orichas. Ces poupées-orichas portent les couleurs du saint qu'elles incarnent. Tout type de poupée peut faire l'affaire, on trouve même des Barbie®-orichas. Ces objets, très domestiques en apparence, pour autant qu'ils reçoivent le même traitement rituel que les autres orichas (libations de sang animal, remplissage 
par des substances magiques), sont, tout comme les soupières, des trompe-l'œil puisqu'ils ne ressemblent pas à des artefacts religieux.

Dans la continuité esthétique de ces objets religieux «domestiques »ou « luxueux », il y a ceux inspirés de l'iconographie catholique. Les orichas sont fortement associés, presque identifiés, aux saints catholiques, comme d'ailleurs l'atteste le nom même du culte : santería provient du mot « santo » (saint), de sorte qu'elle peut être traduite comme « la religion des saints » (littéral. santería signifie « sainterie $»^{2}$ ). Il ne faut donc pas s'étonner si on utilise des effigies de saints chrétiens pour représenter les orichas. Il s'agit là d'un processus de syncrétisation qui a commencé dès la colonisation quand, en 1755, l'évêque de La Havane, Pedro Morell de Santa Cruz a imposé à chaque cabildo de se choisir un saint patron catholique. Selon Marks, ce système d'association entre saints catholiques et orichas a esquissé les contours du « double syncrétisme », africain et catholique (cité par Brown 2003, p. 34). Par exemple, Changó, le dieu de la foudre et du tonnerre, peut être représenté par une statue de plâtre de sainte Barbe.

Ici, l'élément religieux n'est pas dissimulé comme dans le cas des soupières ou d'autres récipients, il est déguisé. En revanche, si l'on y regarde de plus près, on constate que le déguisement est partiel, que les effigies sont personnalisées de telle sorte qu'elles reproduisent l'esthétique surchargée, chère aux santeros. On habillera par exemple l'effigie de sainte Barbe de tenues aux tissus brillants et aux couleurs de l'oricha Changó - blanc et rouge éclatants - et on le complétera d'accessoires n'ayant manifestement qu'un rapport lointain au catholicisme, tels que des bandeaux, des perruques, d'imposants bijoux rituels, on l'entourera d'offrandes d'alcool et de cigares... Parfois, la taille de ces effigies est d'ailleurs supérieure à l'échelle humaine.

La santería/Ifá cultive aussi une autre facette, moins exposée, incarnée dans un ensemble d'objets qui projettent une image moins policée. C'est le cas des avatars des trois orichas «guerriers » (guerreros) : Eleguá (le messager), Ogún (le forgeron) et Ochossi (le justicier chasseur). Ils se présentent sous la forme de récipients en fer, en terre cuite ou en ciment, remplis de pièces métalliques (couteaux, clous, flèches, parfois menottes, pistolets, boules de fer), de pierres, de cauris et parfois de plumes d'oiseaux et de cornes animales. Les Eleguás sont souvent représentés par une tête humanoïde faite de ciment ou à partir d'une noix de coco (Figure 4). Grossièrement modelées, produites de la main même des santeros, aucune de ces effigies n'est manufacturée (même si certaines sont vendues en boutique) - à dessein puisque ces assemblages de puissances doivent évoquer la nature sauvage, la combativité, une Afrique fantasmée en quelque sorte. Nous sommes loin ici de la très " civilisée " soupière. C'est également le cas des objets qui représentent les eggun, les esprits des morts et des ancêtres de la santería et du culte d'Ifá, tout aussi sommaires et bruts.

2. Je remercie l'un des évaluateurs de cet article d'avoir porté cette précision à ma connaissance. 


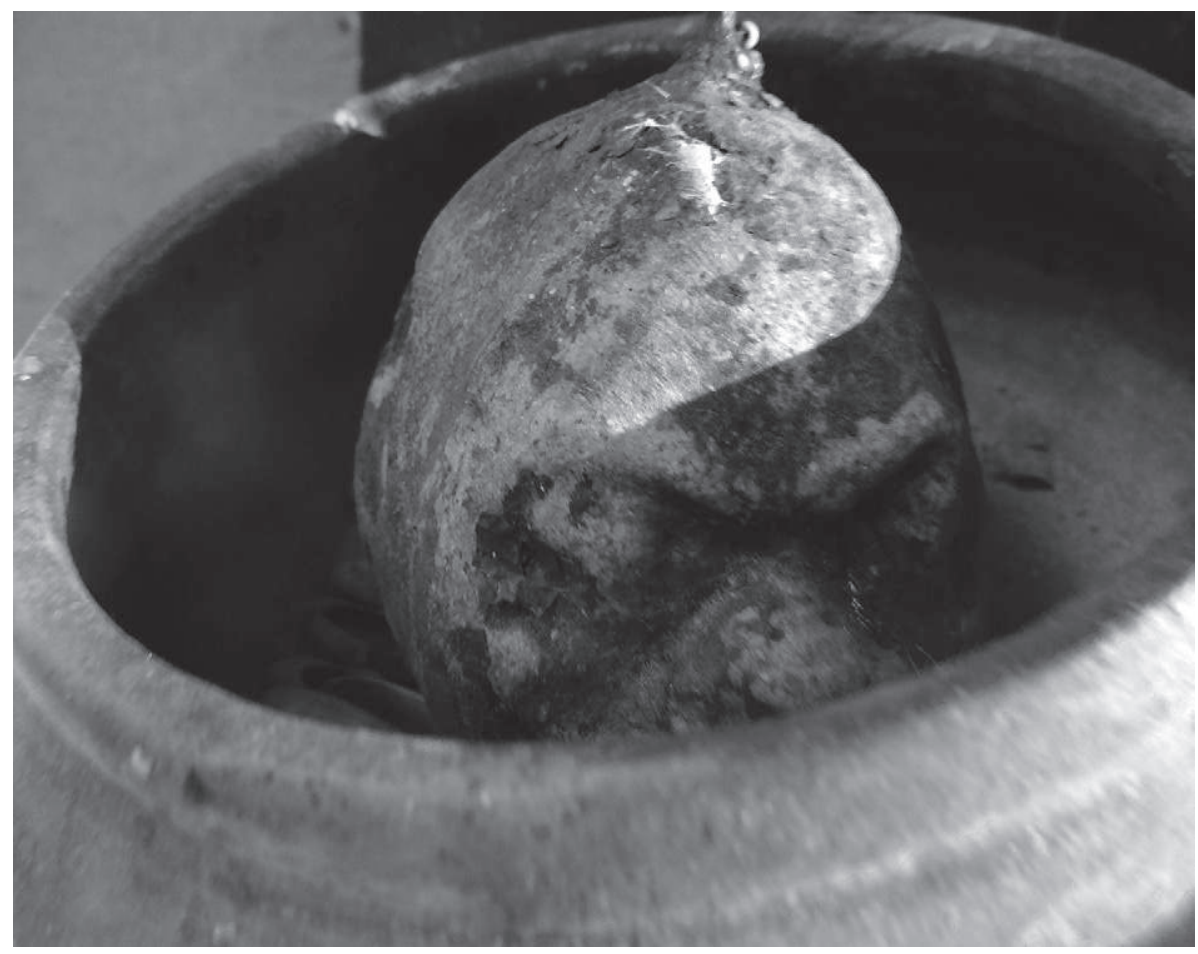

Fig. 4 - La divinité Eleguá de la santería.

Palo monte, sorcier, sauvage, terrestre

Le palo monte a été accusé par les adeptes d'autres cultes d'être le plus perméable des cultes afro-cubains aux influences externes. Or, lorsqu'on examine son esthétique, on constate qu'il est le moins syncrétique d'entre eux. L'examen de ses lieux de culte, les cuartos de fundamento (litt. « pièces de fondation »), en témoigne : leurs espaces sombres, leurs objets énigmatiques, leurs odeurs de putréfaction - tout ici évoque l'univers des morts « obscurs » du palo monte qui « s'alimentent de sang ». Et pour cause, l'intention de leurs architectes est d'y recréer un environnement évocateur d'une nature sauvage, la " wilderness » (Palmié 2002), où s'épanouissent ces morts qu'on nomme nfumbis (Figure 5, page suivante). Les nfumbis sont les véritables protagonistes du culte et les détenteurs du savoir et du pouvoir des paleros. De manière générale, le cuarto de fundamento doit provoquer de l'inquiétude chez le visiteur car ces morts sont des êtres dangereux et capables de tout. Une esthétique morbide le caractérise : on y voit des crânes, des cornes et des pattes d'animaux, des charognards embaumés, des exosquelettes d'insectes et parfois des ossements humains, un tibia, un morceau de crâne laissés à la vue sans compter la croute de sang sec 


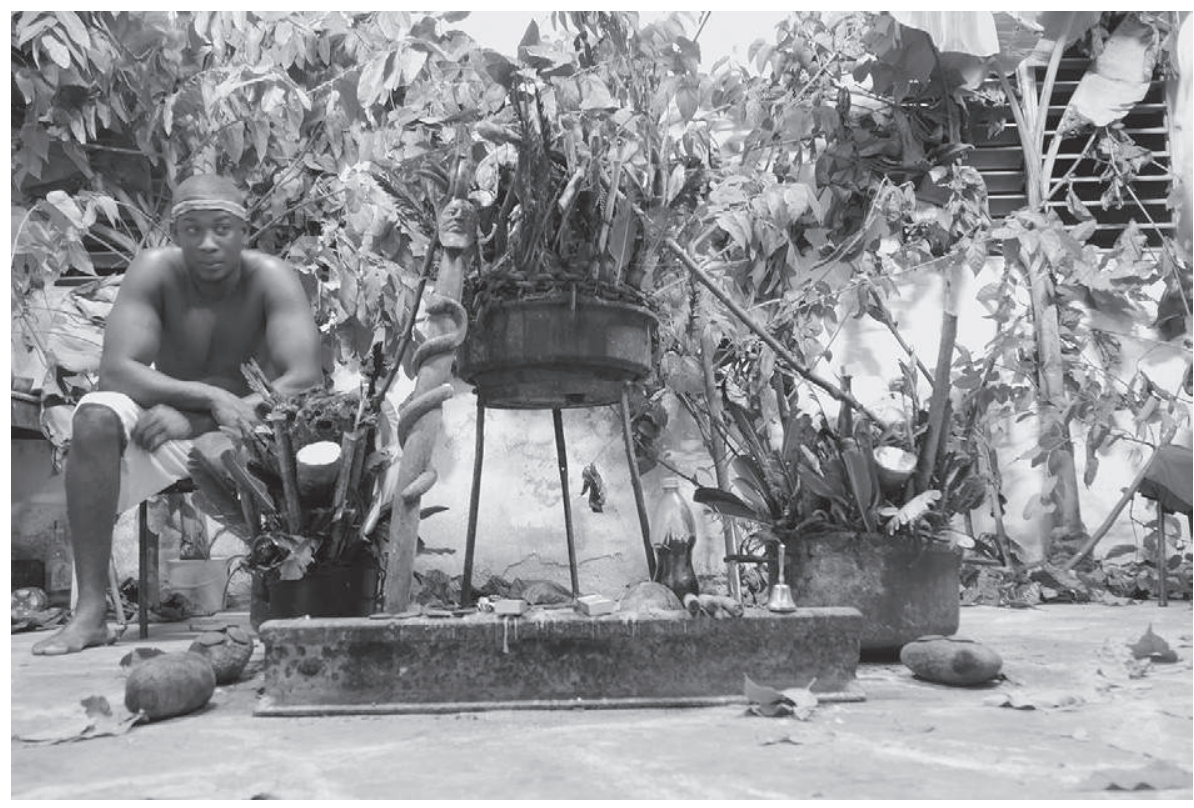

Fig. 5 - Sanctuaire du palo monte.

qui recouvre chacun des objets présents sur l'autel. Nous sommes là bien loin de l'esthétique flamboyante des artefacts immaculés des santeros et des babalaos qui se précipitent après leurs libations pour nettoyer et polir leurs fundamentos.

J'ai déjà écrit sur la nganga, un objet qui abrite/incarne les morts du palo monte et qui se présente comme une synecdoque de l'esthétique palera (Kerestetzi 2016). Elle se matérialise sous forme d'un chaudron qui contient des ossements humains, trouvés et déterrés au hasard des cimetières ou volés dans des ossuaires, ainsi qu'une multiplicité d'autres éléments de la nature (Figure 6). Elle est noircie par le sang sacrificiel et gonflée par tous les ingrédients qui s'accumulent au cours du temps - plumes, ossements, une chaîne, ses « yeux », deux cornes de bœuf terminées par deux morceaux de miroir, une machette, des branches d'arbres nues (palos), des clous et des crochets. Indépendamment de toute médiation symbolique, la nganga expose à la vue les principes mobilisés par le palero : forces de la nature, pouvoir régénérateur du sang versé, agression, mort et mystère, Afrique des puissances antiques. Véritable hybride des forces naturelles, elle n'est pas seulement inspirée de la nature sauvage, elle constitue une tentative de la domestiquer. La nganga est d'ailleurs considérée par les pratiquants comme un monde en miniature, une recréation à petite échelle du cosmos naturel et spirituel.

Les autres objets présents dans un cuarto de fundamento reproduisent 1'esthétique sauvage de la nganga mais de manière plus modeste. Il s'agit des 


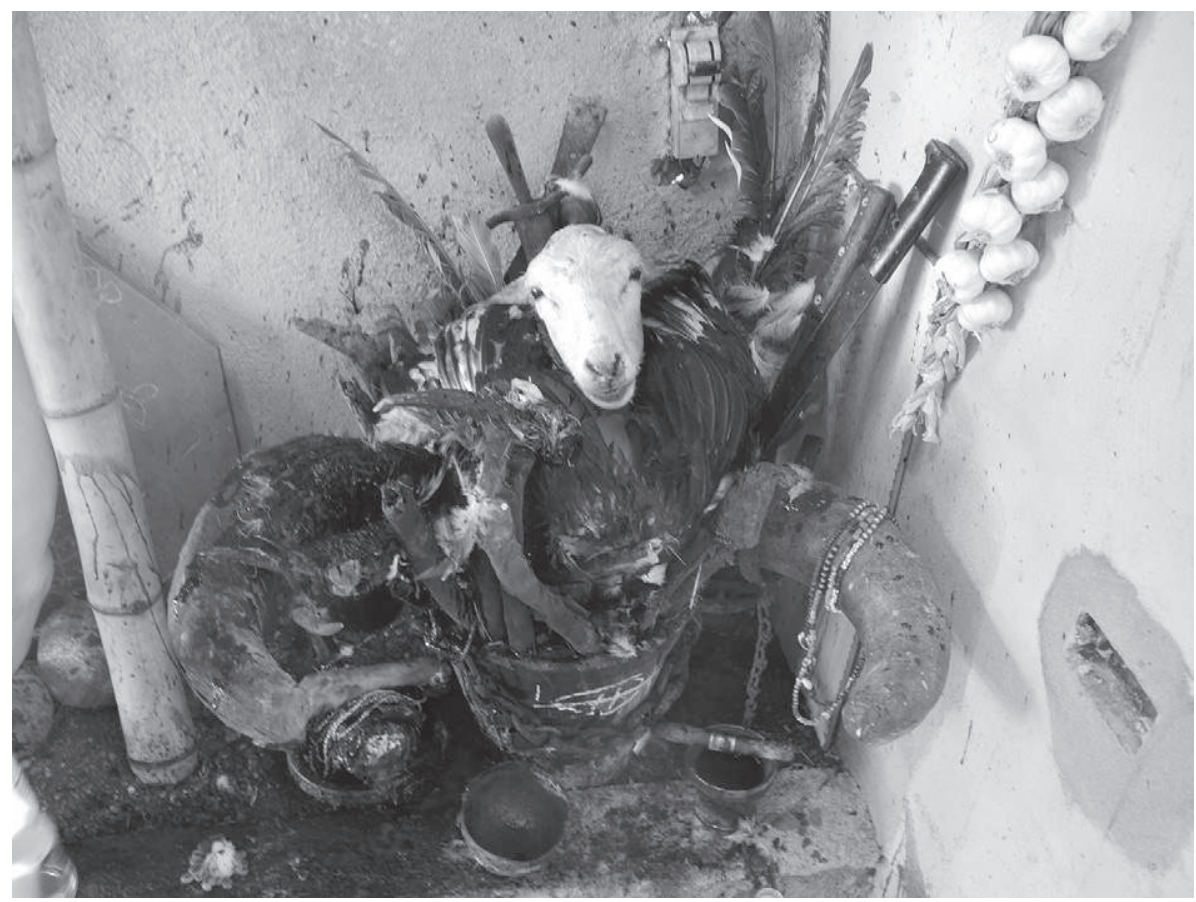

Fig. 6 - La nganga du palo monte après avoir reçu le sang d'une chèvre.

figurations des mpungus, les divinités auxiliaires des morts. De taille plus petite, leurs représentations sont des objets créés par les paleros : ressemblant aux guerriers de la santería, il s'agit d'effigies sommaires en bois ou en ciment, de petits récipients remplis des pierres et de métaux, de noix de coco évidées. Ces artefacts sont bruts, ils ne sont ni sophistiqués ni luxueux comme certains objets de la Ocha/Ifá. Ils sont faits de matériaux rudimentaires, grossièrement taillés, sans préoccupation esthétique.

\section{Le spiritisme : affection, intimité}

L'autel du spiritisme, la bóveda espiritual ou mesa (table) est l'installation religieuse la plus discrète du complexe afro-cubain ${ }^{3}$. Il s'agit généralement d'une simple table couverte d'une nappe blanche, sur laquelle sont posés des photos de parents décédés, des verres d'eau, des vases fleuris, des poupées

3. Si j'inclus le spiritisme dans l'ensemble des cultes afro-cubains, c'est parce qu'il constitue bel et bien une composante importante de certains parcours religieux des initiés aux cultes afro-cubains et parce qu'il a intégré dans sa pratique plusieurs éléments « africains », tels que la possession par des esprits congolais ou encore l'usage du rhum et des cigares (voir aussi Argyriadis 1999). 


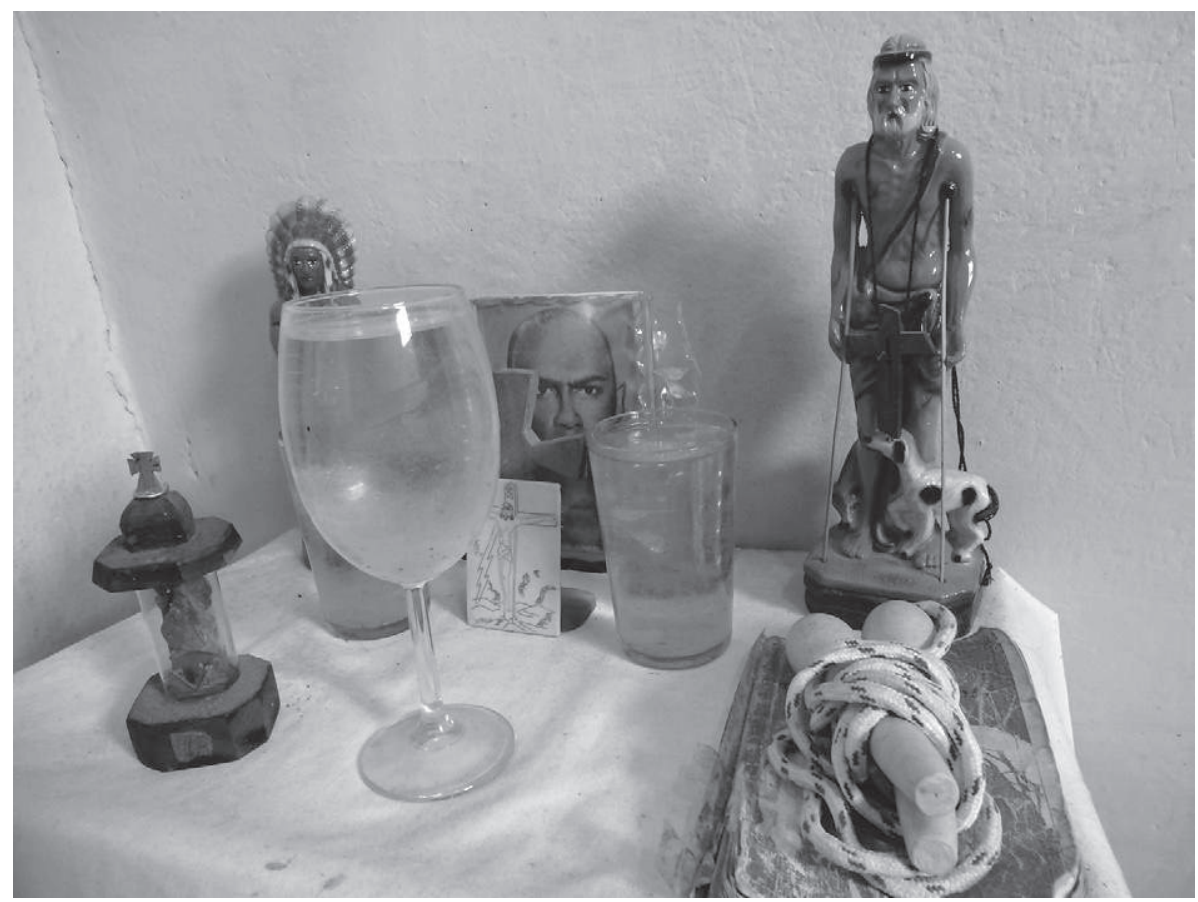

Fig. 7 - Autel du spiritisme.

pour enfant ; parfois y figurent aussi des objets ayant appartenu aux défunts, tels qu'une pièce d'identité ou un bracelet. Sans la présence d'un crucifix, rien ne trahirait pour un œil non averti la fonction religieuse de l'ensemble, tout du moins pour les plus minimalistes d'entre eux (Figure 7).

Les bóvedas rendent présente la " commission » (comisión) d'esprits qui protègent et accompagnent l'individu depuis sa naissance. Pour le spiritisme cubain, chaque personne est protégée par plusieurs « anges gardiens » (ángeles guardianes) qui peuvent être des esprits de parents mais aussi de Tsiganes, d'Indiens, d'Africains, d'artistes, de riches commerçants, etc. Chacun d'entre eux peut figurer sur une bóveda; l'artiste peut prendre la forme d'un instrument de musique, la Tsigane celle d'une poupée vêtue à l'andalouse... Certains espiritistas (spirites) choisissent de ne représenter sur leur « table » que les esprits qu'ils considèrent comme les plus proches, d'autres peuvent au contraire agrandir leur installation, en la faisant déborder sur les meubles ou les murs voisins pour inclure le plus d'esprits possible (Figure 8).

Par leur caractère très personnel donc, les bóvedas sont de véritables « soi extériorisés », ou des personnalités « disséminées » (Gell 1998) dans l'espace. Les esprits qu'ils représentent sont censés refléter chacun un aspect de la personnalité 


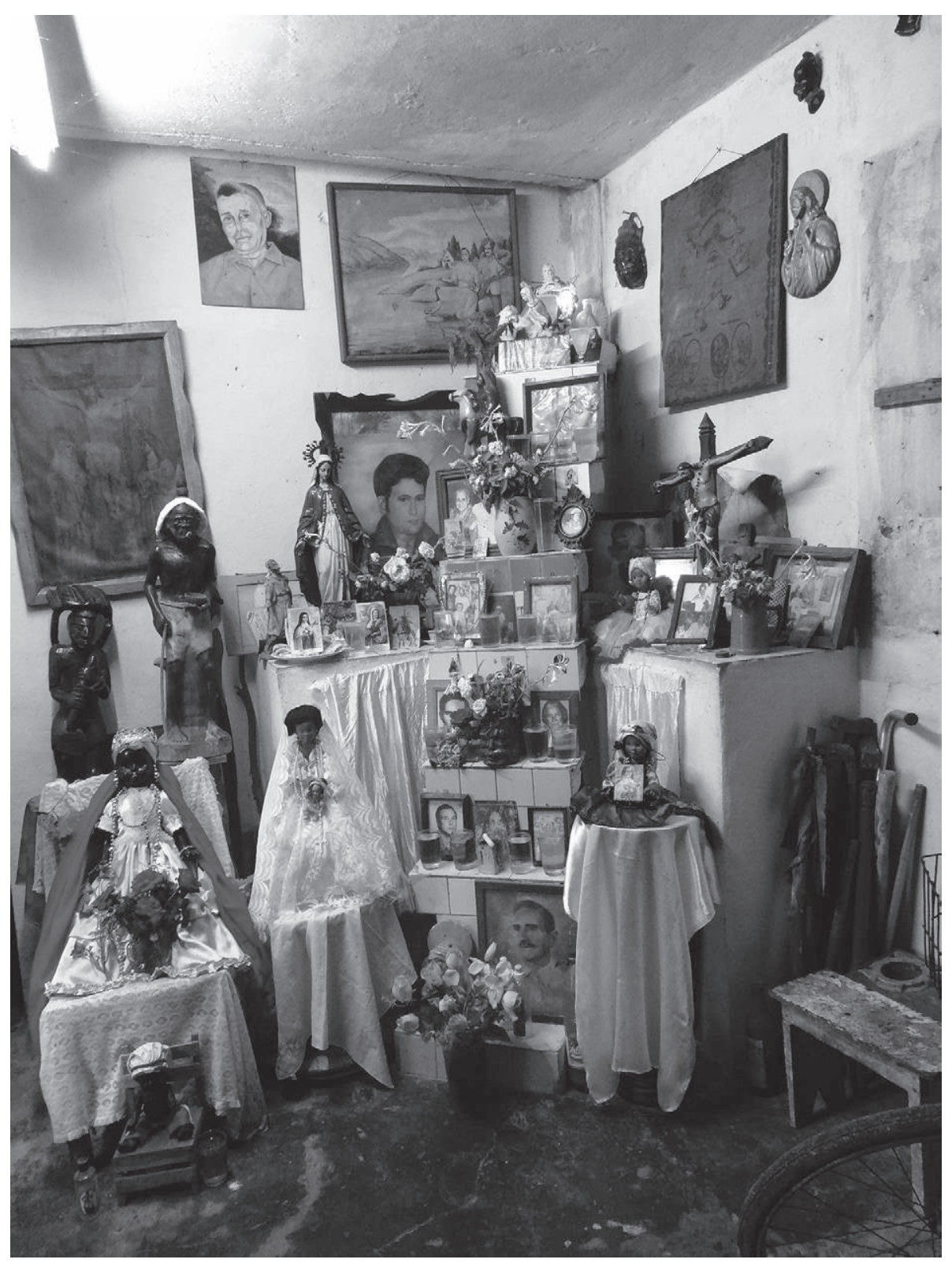

Fig. 8 - Autel du spiritisme. 
de leur possesseur. Dans un guide de la pratique spirite, le Manual del Espiritista (cité par Espírito Santo 2009, p. 177), l'auteur anonyme, spirite-lui-même, livre une interprétation intéressante : « Le montage de la bóveda espiritual est une chose très variable, puisque plusieurs facteurs ou objectifs rentrent en compte dans ce processus. Chaque bóveda est en elle-même une représentation spirituelle-artistique de chaque individu », une charte de personnalité ou une marque de soi. Mais elle est aussi, pour son possesseur, un objet de mémoire et d'une histoire familiale, un objet d'affection, reflétée dans les yeux des parents décédés.

Le caractère idiosyncrasique des bóvedas les rend facilement « assimilables » par les membres des autres cultes afro-cubains qui, sans être spirites dans le plein sens du terme, peuvent trouver utile de profiter de la protection des morts spirites. Il est fréquent qu'un adepte de la santería soit incité par un santero ou un babalao à se doter d'une bóveda afin que les esprits du spiritisme lui « ouvrent les chemins » du bonheur et rendent le travail de son oricha gardien plus facile. Les bóvedas se présentent ainsi comme des produits d'une philosophie existentielle qui combine des fragments conceptuels et matériels hétérogènes d'une manière très personnelle.

\section{L'écologie des objets : principes généraux}

La maison-temple afro-cubaine peut être vue comme un écosystème. Elle abrite une multitude d'esprits, d'entités et d'êtres humains, autant d' " espèces » différentes qui vont interagir les unes avec les autres et vont devoir s'adapter à leur milieu ambiant, en fonction de contraintes internes et externes. Certaines entités peuvent être considérées comme prédatrices, d'autres ont une faculté d'adaptation plus large, d'autres encore ne peuvent s'épanouir que dans une niche très réduite. L'emplacement des différents objets religieux dans une maison de spiritualité mixte est d'une certaine manière une projection architecturale des différentes forces en présence. Ces forces ont une expression concrète : ce sont elles qui dirigent la main de l'habitant de la maison lorsque ce dernier installe un nouvel objet dans son habitat. Elles sont idiosyncrasiques et multifactorielles, et sont le produit pour partie de prescriptions religieuses, d'une esthétique personnelle, de facteurs topologiques comme des contraintes architecturales et de dynamiques familiales, de jeux de pouvoirs, de rivalités et d'affinités.

Principes généraux des emplacements des objets et de la construction de l'espace domestico-rituel

La construction de l'espace domestico-rituel suit deux exigences : premièrement, isoler la maison du monde extérieur et de ses mauvaises influences, deuxièmement, agencer son intérieur pour se plier aux exigences cosmologiques et pour éviter les frictions entre divinités. 
Pour protéger leur maison, les pratiquants emploient différentes techniques : amulettes apotropaïques qui encerclent le terrain, divinités qui guettent derrière les portes d'entrée, croix chrétiennes qui décorent les seuils et les portes. D'ordinaire, une maison santera est protégée du monde extérieur par la présence d'amulettes-barrages implantées aux quatre coins du terrain. Ces guardianes (gardiens), peuvent prendre la forme de crampons de rail ou de fers à cheval qu'on enfonce simplement dans le sol, ou d'amulettes qui pendent derrière la porte d'entrée. Parfois, il s'agira d'objets plus sophistiqués. Certains enterrent des amulettes devant leur porte d'entrée, ce qui implique parfois de casser et de refaire le ciment ou la pierre du seuil de la maison, y compris quand ce dernier se trouve sur la voie publique (Figure 9, page suivante). Les paleros aussi protègent leur habitat du monde extérieur, en enterrant des amulettes, les masangos, aux quatre coins de leur terrain et en traçant des signes magiques (on y reviendra). Quant aux spirites, ils peuvent asperger la maison d'eau bénite, ficher des croix chrétiennes sur les seuils, installer l'effigie d'un esprit bien en face de la porte d'entrée. La maison elle-même doit être préparée pour accueillir de nouveaux venus spirituels. Lorsque, par exemple, un santero ou un babalao veut installer de nouveaux objets rituels, il doit purifier rituellement sa maison - « pour que les orichas se rendent compte qu'ils vivent dans la maison, pour leur "donner entrée dans la maison" (darle entrada en la casa) », nous dit un santero.

La deuxième exigence architecturale est infiniment plus complexe à gérer pour les pratiquants puisque nous ne sommes plus en situation de simple dialectique - la maison et le dehors - mais face à une multiplicité d'artefacts religieux issus de religions différentes, le tout interagissant dans un espace architectural singulier. Dans certaines maisons, on doit faire coexister les divinités africaines et prestigieuses de la santería, les morts obscurs et malins du palo monte, les morts familiaux et épurés du spiritisme, ainsi que le Christ ou autres saints catholiques. À cette situation déjà complexe s'ajoutent en outre les incompatibilités entre entités de la même religion. Par exemple, le babalao Fernando nous dit qu'Oyá, la déesse de la mort a, dans la mythologie santera, pour ennemie jurée Yemayá. Pour éviter les conflits, il veille donc à ce que leurs soupières respectives soient tenues éloignées l'une de l'autre.

Pour les membres de la maisonnée, chaque objet religieux de l'habitat, parce qu'il est considéré comme intentionnel, est, à lui seul, un opérateur de spatialisation, un « sous-espace centré » (Hanks 1996, p. 180) et agissant. Dans ce type de maison - pluri-centrique donc -, il faut savoir faire preuve d'une grande inventivité pour mettre en relation ou pour séparer ses sous-espaces. Car dans ce domaine, au mieux les religions ne donnent que de grands principes directeurs peu adaptés à des situations nécessairement uniques.

Prenons l'exemple d'une maison dans laquelle habitent des pratiquants du palo, de la santería, du spiritisme et du catholicisme. Quand on entre dans une telle maison, les premiers objets qu'on perçoit appartiennent d'habitude à l'univers 
Katerina KeRESTETZI

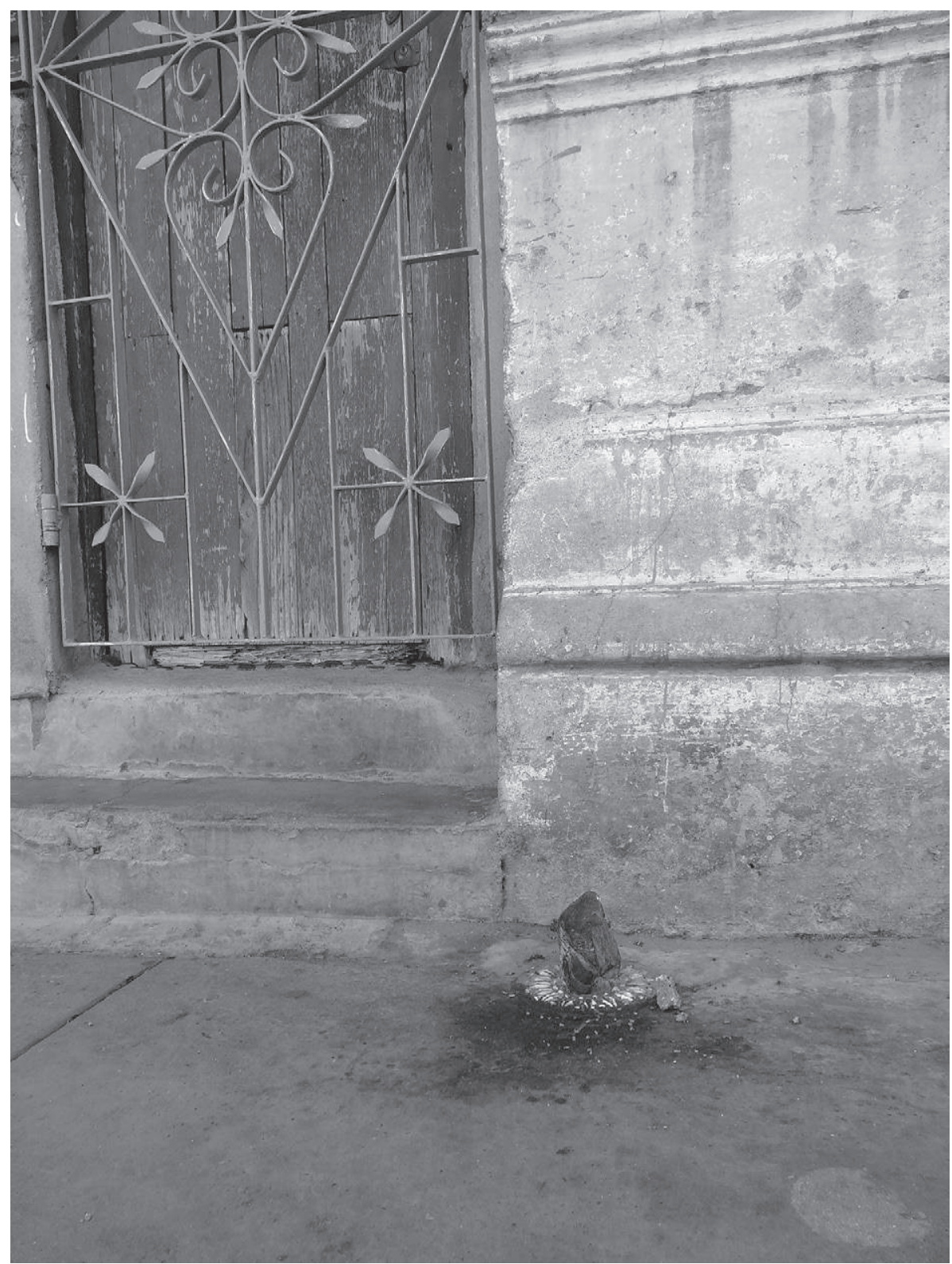

Fig. 9 - L'objet fixé sur le trottoir est un oricha « gardien » de la maison. 
catholique/santero/Ifá. Parfois, ce sont des effigies monumentales, à taille humaine, de saints chrétiens (en réalité des orichas) qui accueillent le visiteur. Richement peintes, elles font la fierté de leurs propriétaires et ont été achetées en boutique à un prix élevé. Souvent, elles sont disposées de façon à pouvoir être perçues depuis la rue. D'autres symboles proprement chrétiens - croix et crucifix, petites effigies de saints catholiques, posters du Christ - peuvent décorer le salon et l'entrée. L'installation de ces objets dans les pièces visibles de la maison obéit plutôt à des critères esthétiques. Parce qu'ils sont « beaux » et propres, ils sont exposés à la vue de tous. Bien sûr, le critère esthétique est précontraint par les représentations théologiques sur les dispositions des entités mêmes. Puisque Jésus est censé apporter la paix et l'amour, il est logique d'installer son poster dans un espace de sociabilité comme le salon.

Or c'est parfois le type même du support qui influence le choix de son emplacement - il suffit de songer aux soupières de la santería. Ces soupières en porcelaine (les soperas) des orichas sont d'ordinaire exposées bien en vue, comme s'il s'agissait d'objets de grande valeur : sur une étagère au beau milieu du salon ou d'un hall et le plus souvent dans une élégante armoire vitrée, le canastillero (Figure 10, page suivante). Certains peuvent installer leurs soupières dans une bibliothèque ou dans un placard ouvert; 1'essentiel est qu'il y ait des étagères de façon à former des niveaux ${ }^{4}$. Les niveaux du support sont censés refléter un ordre cosmique mais, dans la majorité de cas, ils reflètent la vision singulière d'un initié de cet ordre supposé : généralement, c'est Obatalá, l'oricha le plus vieux et le plus sage qui est posé sur l'étagère la plus haute, en bon juge de ce qui passe dans la maison et dans la religión; certains peuvent installer leur « ange gardien », c'est-à-dire leur oricha protecteur, au deuxième plus haut niveau mais d'autres préfèrent lui trouver une place individuelle, loin du meuble collectif, pour exposer ainsi le rôle majeur qu'il tient dans leur vie ; le troisième niveau peut être occupé par Yemayá et Ochún, mais il arrive que des initiés changent la position de leurs récipients, afin d'éviter que l'aché, l'énergie spirituelle et vitale, sorte de mana cubain (Holbraad 2007) qui circule entre eux ne stagne. Il se peut aussi qu'un canastillero soit partagé entre plusieurs santeros de la famille. Dans ce cas de figure, la disposition des artefacts peut tout simplement être inspirée des dynamiques et des rapports familiaux qui deviennent du même coup des composantes cosmologiques. Par exemple, les soupières de Pedro sont au même niveau que celles de sa première et unique épouse ; chez Izrael, l'armoire est divisée verticalement en deux parties égales : l'une est réservée à

4. Chaque objet rituel de la santería est personnel. Chaque initié est accompagné par plusieurs orichas matérialisés : son principal qui est son « ange gardien », les trois « guerriers » ainsi que les quatre orichas qui accompagnent « obligatoirement» un aspirant (Gobin 2012, p. 80), à savoir Obatalá, Changó, Yemayá et Ochún. Au besoin, il peut aussi se doter de l'intégralité du panthéon oricha. Ces objets sont habituellement logés chez leur dépositaire mais il arrive que ce dernier, faute d'espace, les confie à son initiateur(trice). 
Katerina KeRESTETZI

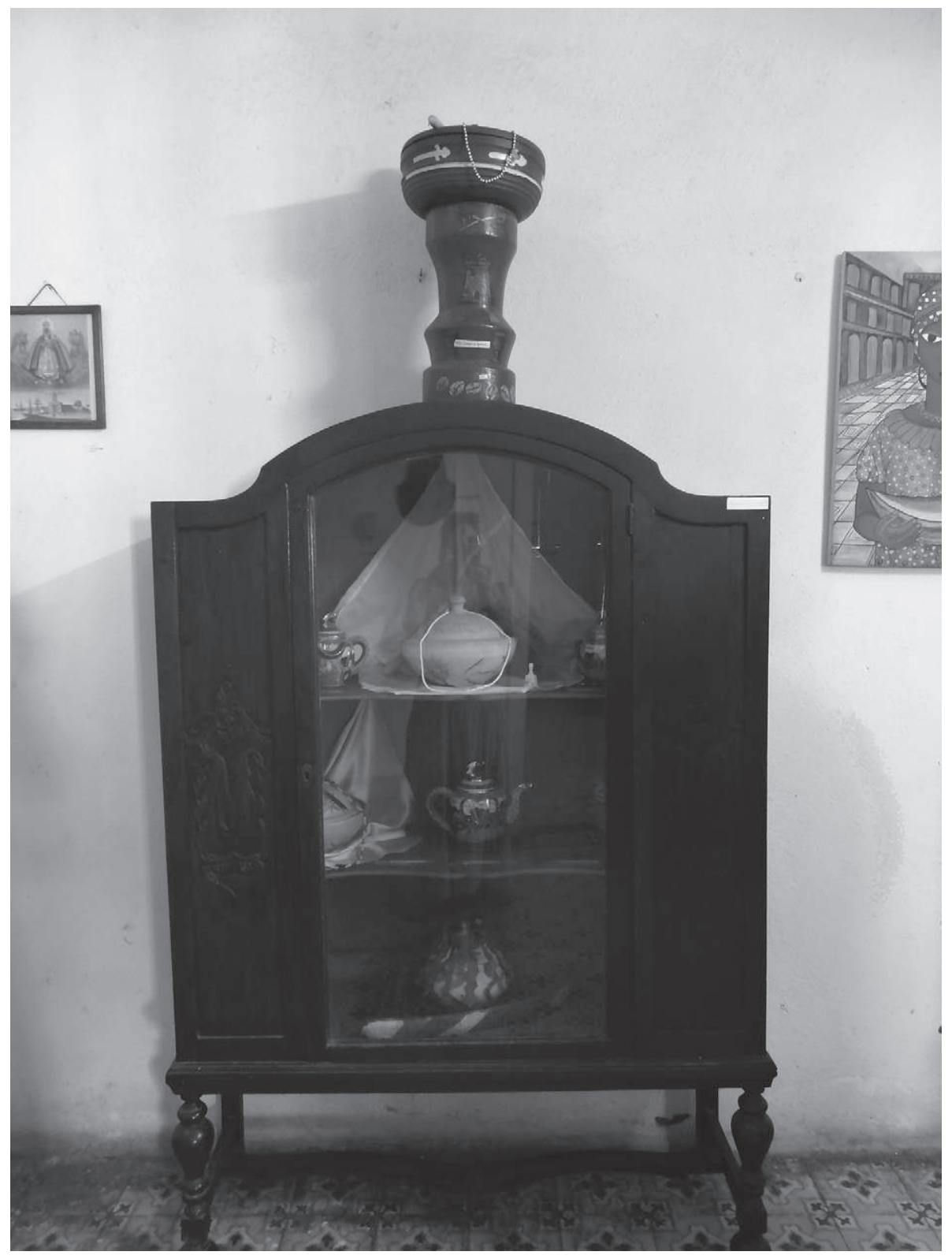

Fig. 10 - Le canastillero. 
ses soperas et à celles de ses enfants, issus d'un premier mariage, et l'autre aux soperas de son épouse et de ses enfants, eux aussi issus d'une union précédente. Comme le dit Izrael, " les siens avec les siens et les miens avec les miens ». Pour ce qui le concerne, il y a séparation mais pas hiérarchie divine : chacune des parties du meuble loge les orichas de manière aléatoire.

Dans la spatialité santera/Ifá, la verticalité est une notion bien complexe. Le haut et le bas peuvent parfois constituer des catégories morales ou hiérarchiques - le haut étant associé au ciel et au dieu suprême, Olofi, et le bas, à la terre, aux hommes. Par exemple, pour illustrer auprès de son jeune fils la valeur supérieure de sa divinité préférée, Orula, un babalao a placé son fundamento au sommet d'un haut placard afin que son fils se dise « mon Orula vit là-haut, mon Orula est grand ». La hauteur de l'emplacement joue ici le rôle de signifiant, elle fait partie du message communiqué par l'entité ou, autrement dit, elle est indice de sa puissance. Or, le plus souvent, on constate que le haut et le bas constituent simplement des catégories d'action et d'identification. Certains dieux sont tout simplement plus terriens que d'autres et n'atteignent leur plénitude que lorsqu'ils sont en contact avec la terre. C'est le cas des guerreros, les orichas « guerriers », qui sont généralement posés à même le sol. Les guerreros sont des dieux du bas. Et cela ne désigne aucunement une position d'infériorité par rapport aux autres orichas: d'ailleurs Eleguá, l'oricha qui « ouvre et ferme les chemins » des initiés est toujours le premier à être salué rituellement. Pour les santeros et les babalaos, mais cela vaut aussi pour les paleros, plus une entité est proche de la terre, plus sa capacité d'action concrète s'accroît. $\mathrm{Au}$ sein de l'habitat, les guerreros sont d'ailleurs très actifs car ils participent de manière spontanée à la protection de l'initié et de sa demeure des malheurs et des esprits malins. Cependant, l'agencement des fundamentos des guerriers au sein de l'habitat atteste de leur faible compatibilité avec la domesticité et la vie parmi les humains : ils sont posés à des endroits peu visibles de la maison qui sont rarement investis d'une activité sociale - derrière les portes (celle de l'entrée notamment), derrière un canapé, dans une cuisine, au fond d'un placard (Figure 11, page suivante). Je ne peux pas affirmer que cette disposition « dans les ténèbres 》soit une conséquence directe de leur esthétique inquiétante et d'une volonté délibérée de les soustraire aux regards. Mais ce qui est certain est que leur emplacement dans ces zones obscures les enveloppe littéralement d'une aura liée au danger qui les entoure et rend ainsi manifeste leur capacité à s'opposer à de potentiels ennemis.

L' « esthétique-frontière » de l'environnement du palo monte atteste, quant à elle, une répulsion du monde socialisé qui s'harmonise difficilement avec l'espace de la famille. D'ailleurs, d'un point de vue théologique, les nfumbis sont, parmi les entités spirituelles afro-cubaines, celles qui sont le moins sociables. Ils ne peuvent ni supporter la présence d'agents spirituels exogènes, ni la compagnie des humains (à la notable exception de leur palero). Dans la maison, la 


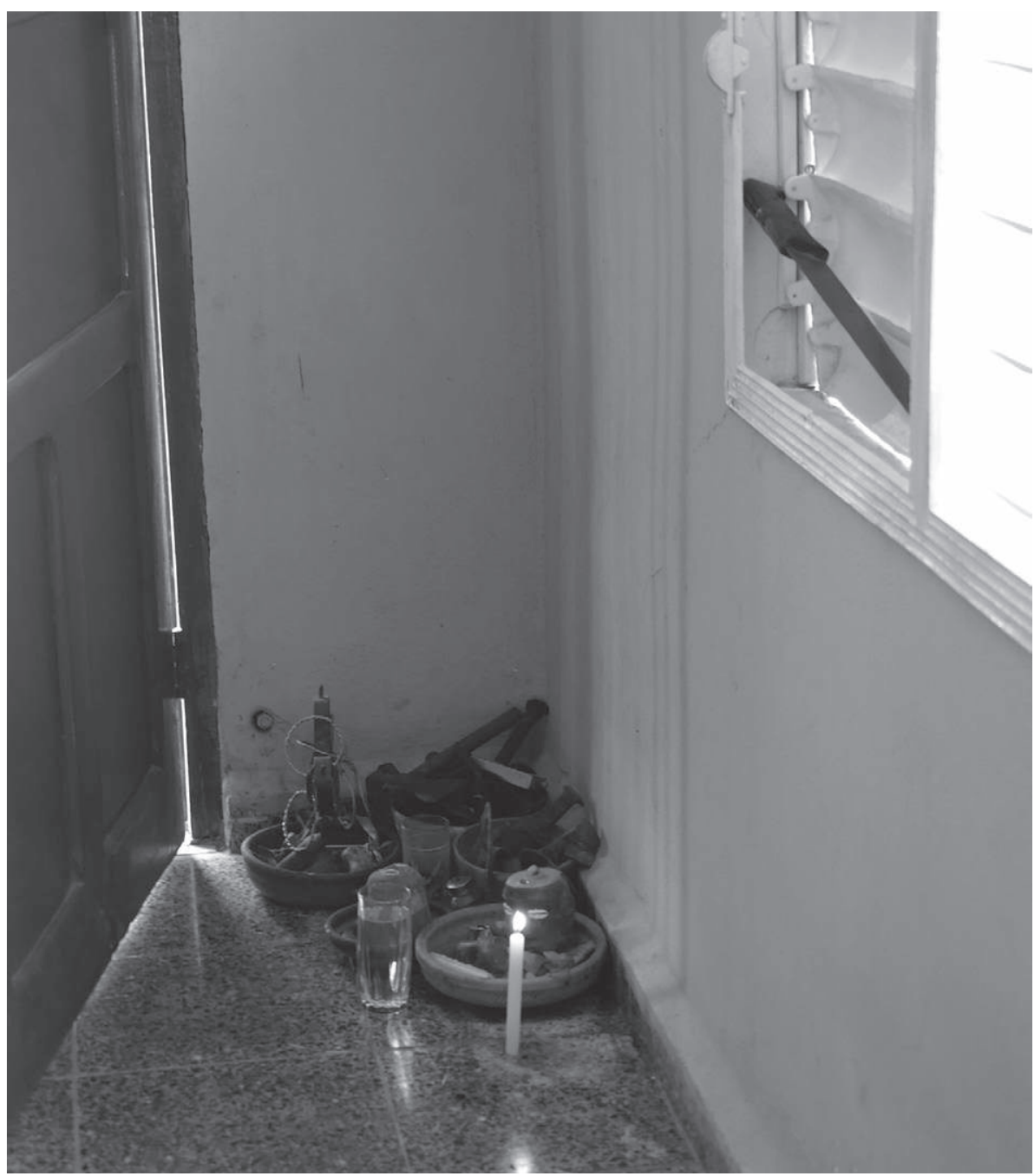

Fig. 11 - Les guerriers de la santería installés derrière la porte d'entrée de la maison.

topographie du culte témoigne de sa nature sauvage et asociale. En général, le palo est logé à l'écart des parties habitées, de préférence dans une cabane en bois sommaire installée dans la cour. De plus, les nfumbis sont bien plus capricieux et vicieux que les orichas de la santería et peuvent constituer un véritable danger pour les proches du palero. Il s'agit généralement d'esprits issus de personnes moralement douteuses, de criminels parfois, qui n'ont pas de scrupules à s'en prendre à ceux qui les importunent, famille du pratiquant inclue. La cohabitation nfumbis-humains est problématique et contraignante pour la vie « profane » d'une 
maisonnée. Le palo monte impose d'ailleurs plusieurs règles et restrictions aux habitants de la maison : interdiction aux femmes de s'approcher des ngangas pendant leur menstruation, interdiction d'avoir des relations sexuelles devant les nfumbis ou de les visiter sans s'être lavé après l'acte sexuel.

Dans l'espace qui leur est dédié, le cuarto de fundamento, les artefacts rituels sont posés à même le sol, voire, dans le meilleur des cas, en contact direct avec la terre. D'ailleurs, certains objets y sont parfois enterrés. Il peut s'agir d'une nganga puissante qui relie ainsi directement les esprits du cuarto aux morts qui vivent sous terre, aux insectes et à l'écosystème souterrain qui a une grande signification symbolique pour les paleros. On voit ainsi s'opposer ce principe d'agencement horizontal à la verticalité des constructions santeras. C'est une image spatiale de la cosmopolitique de chaque culte : dans le palo, cette horizontalité renvoie à un principe d'égalité entre les êtres de ce monde ; dans la santería, c'est la supériorité des divinités qui est exprimée verticalement.

L'emplacement des autels spirites, comme leur esthétique, est très variable : pas de règles pour l'installation des bóvedas, elles peuvent être disposées aux côtés des orichas de la santería, dans l'espace des morts du palo, dans une chambre à coucher, au milieu d'un couloir... tout dépend finalement de la place que les pratiquants décident d'accorder aux morts spirites dans la vie de leurs maisons-temples.

Le spiritisme afro-cubain initie une réflexion intéressante sur des questions de syncrétisme. D'un point de vue théologique, certains de ces principes doctrinaux, largement transmis dans les cercles des pratiquants des religions afro-cubaines, ont servi de levier pour casser certaines frontières religieuses, notamment en incitant à penser les esprits et divinités de toute origine comme faisant partie d'un même continuum spirituel (Palmié 2002 ; Espírito Santo 2015) : les orichas peuvent être vus comme des esprits exceptionnellement puissants, les anges gardiens du spiritisme comme des esprits épurés des attaches matérielles, tandis que les nfumbis des paleros seraient des esprits très matérialistes. On ne doit donc pas s'étonner de voir figurer sur la bovéda d'un spirite qui pratique la santería, un collier de perles ou une poupée vêtue des couleurs de son oricha protecteur, comme si celui-ci était un membre à part entière de sa commission d'anges gardiens. Les bóvedas offrent une surface assez neutre sur laquelle peuvent s'inscrire les éléments déterminant l'identité religieuse du pratiquant. Dans le fond, ces tables offrent une image matérielle de ce glissement ontologique caractéristique du syncrétisme afro-cubain.

\section{Adaptation spatiale et techniques de compartimentation}

La cohabitation religieuse repose tout d'abord, et peut-être sans surprendre, sur des processus de distanciation des cultes au sein de la maison. Parfois, on divise clairement les espaces dédiés à chacun d'entre eux : le salon pour les autels 
de la santería, l'entrée et le hall pour les symboles chrétiens, le couloir pour la table spirite, la cour pour la cabane du palo. C'est là une illustration littérale de la fameuse notion de "syncrétisme en mosaïque » de Bastide (1967) qui peut être entendue pour l'occasion aussi bien comme une métaphore que comme la description d'une réalité spatiale. Or quand l'espace vient à manquer, d'autres méthodes de compartimentation peuvent être employées. Ainsi, le palero qui ne dispose pas d'un espace extérieur pour loger ses ngangas doit trouver une autre manière de séparer ses morts du reste de la maison. La meilleure option possible est de consacrer une pièce de l'habitat à leur usage exclusif. Les moins bien lotis peuvent se contenter de l'isoler visuellement, en l'installant dans un placard qui restera fermé dans les moments d'inactivité ou, pire encore, en la couvrant d'un simple tissu. Ils peuvent cependant aussi les isoler au moyen de dessins magiques, nommés firmas. Les firmas sont des dessins rituels réalisés à la craie. Leur fonction est de canaliser l'action des différentes divinités et esprits qui participent au travail de palo. Par exemple, quand la nganga est par nécessité installée dans une chambre à coucher, pour que le couple qui l'occupe puisse avoir des relations sexuelles « sans offenser » les morts, il peut séparer symboliquement leur autel en l'encerclant d'une firma tracée au sol (Cabrera 1986, p. 140) qui va leur obstruer la vue. Ces graphismes ont la particularité d'être orientés dans l'espace, leur directionalité s'exprimant par les nombreuses flèches ou lignes dont elles sont composées. Lorsqu'ils sont tracés sur les portes et fenêtres, ils remplissent une fonction apotropaïque en éloignant les esprits malveillants des parties les plus exposées de la maison (Figure 12). Ces dessins marquent non seulement le passage d'un espace à un autre (de la rue à la maison lorsqu'ils sont tracés sur un seuil, ou d'un lieu du palo à l'espace habité...), mais le rendent également infranchissable.

Lorsque les techniques rituelles font défaut et que l'architecture ne s'y prête pas, le compartimentage des religions fait appel à l'inventivité des pratiquants. Le dessous d'un lit peut faire office d'abri pour des entités solitaires. Le dessus d'un réfrigérateur ou d'une armoire peut devenir le «trône » des divinités les plus « célestes ». Parfois, on fera d'un drap suspendu une cloison, d'une table de cuisine, un autel. Les coins des pièces et le revers des portes sont des spots de prédilection pour plusieurs entités ; ils permettent de former des sous-espaces intéressants dans l'espace global de la maisonnée (Figures 13-15, pages suivantes)... Ici, la distinction entre sens religieux et sens pratique est par nécessité ambiguë.

Parfois, « l'acclimatation » des divinités au sein de l'habitat requiert de véritables sacrifices. Par exemple, les morts de la santería, les eggun, ne font pas bon ménage avec les intérieurs. Leur " coin », le rincón de eggun, est idéalement installé dans la cour de la maison ou, à défaut, sur un balcon, à côté de canalisations (Gobin 2012, p. 436). Dans un appartement santero de Cienfuegos, on a créé un espace pseudo-extérieur pour les installer, en réaménageant une cuisine 


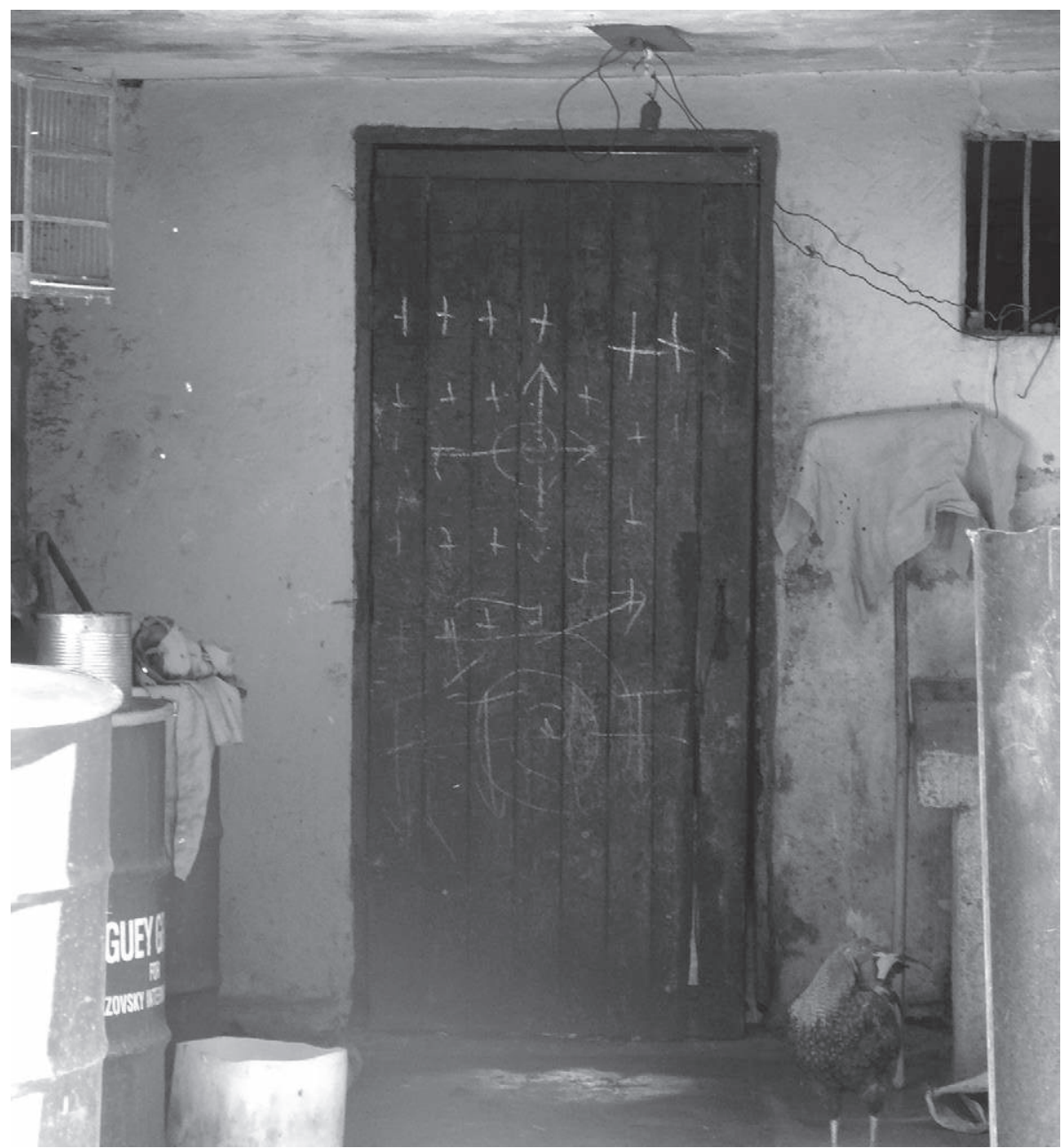

Fig. 12 - Firmas apotropaïques, tracées sur la porte d'une maison du palo monte.

qui s'ouvrait sur une large fenêtre sans volets ni vitres, en espace exclusivement rituel (on y effectue aussi des sacrifices ou prépare des plats rituels...). Ce réaménagement au profit de l'épanouissement de ces esprits associables, dans un espace à part, a obligé la famille à condamner une pièce d'un appartement déjà petit pour construire une nouvelle cuisine à usage quotidien (Figure 13). Domestiquer l'indomesticable a un coût parfois. C'est également le cas d'Ossain, la divinité la plus sauvage et « sorcière » des babalaos, maître de la forêt et de la pharmacopée, qui demande systématiquement à être installé en retrait de l'habitat, dans un patio ou une cour. 


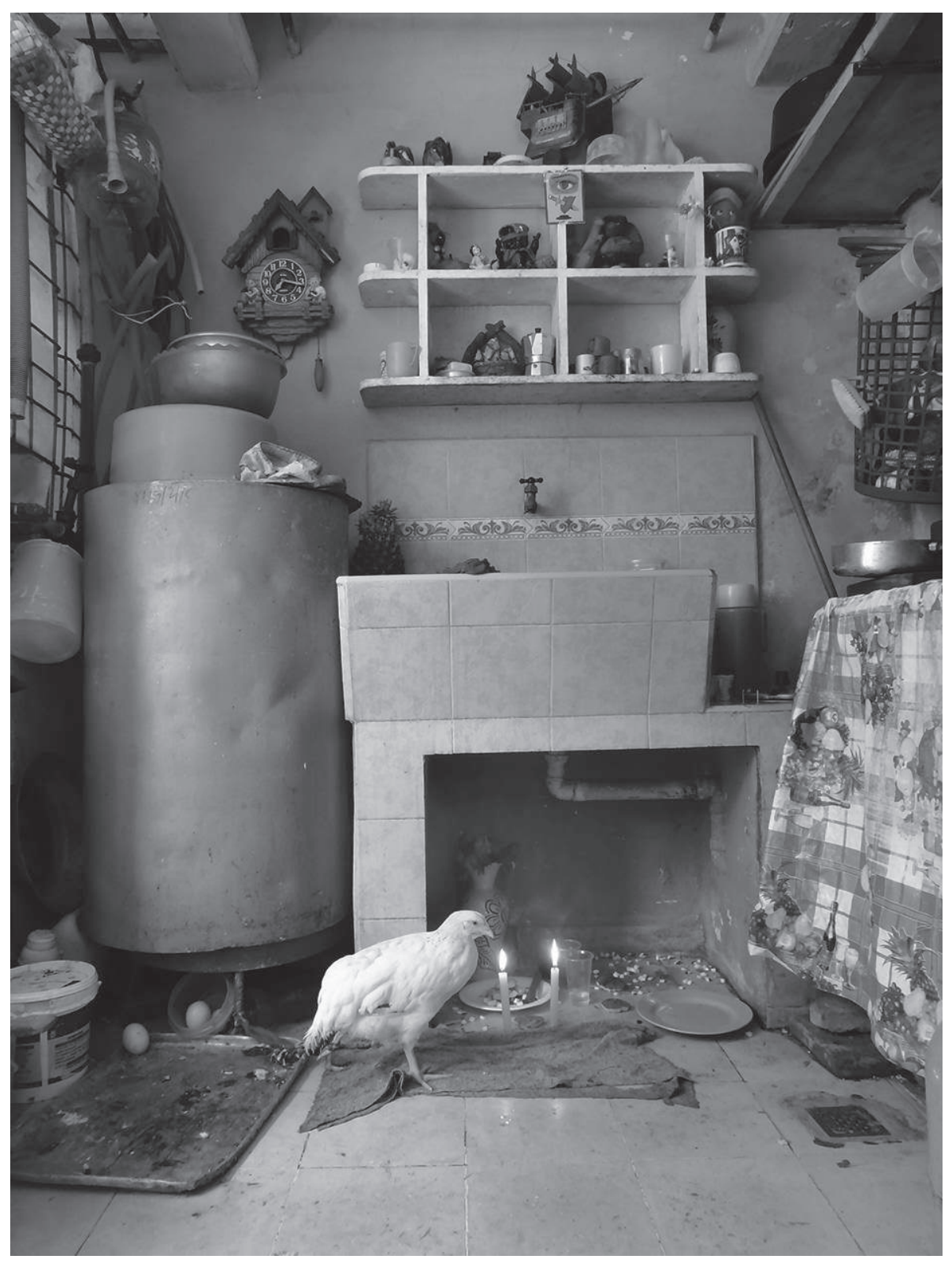

Fig. 13 - Cuisine réaménagée en espace rituel. 


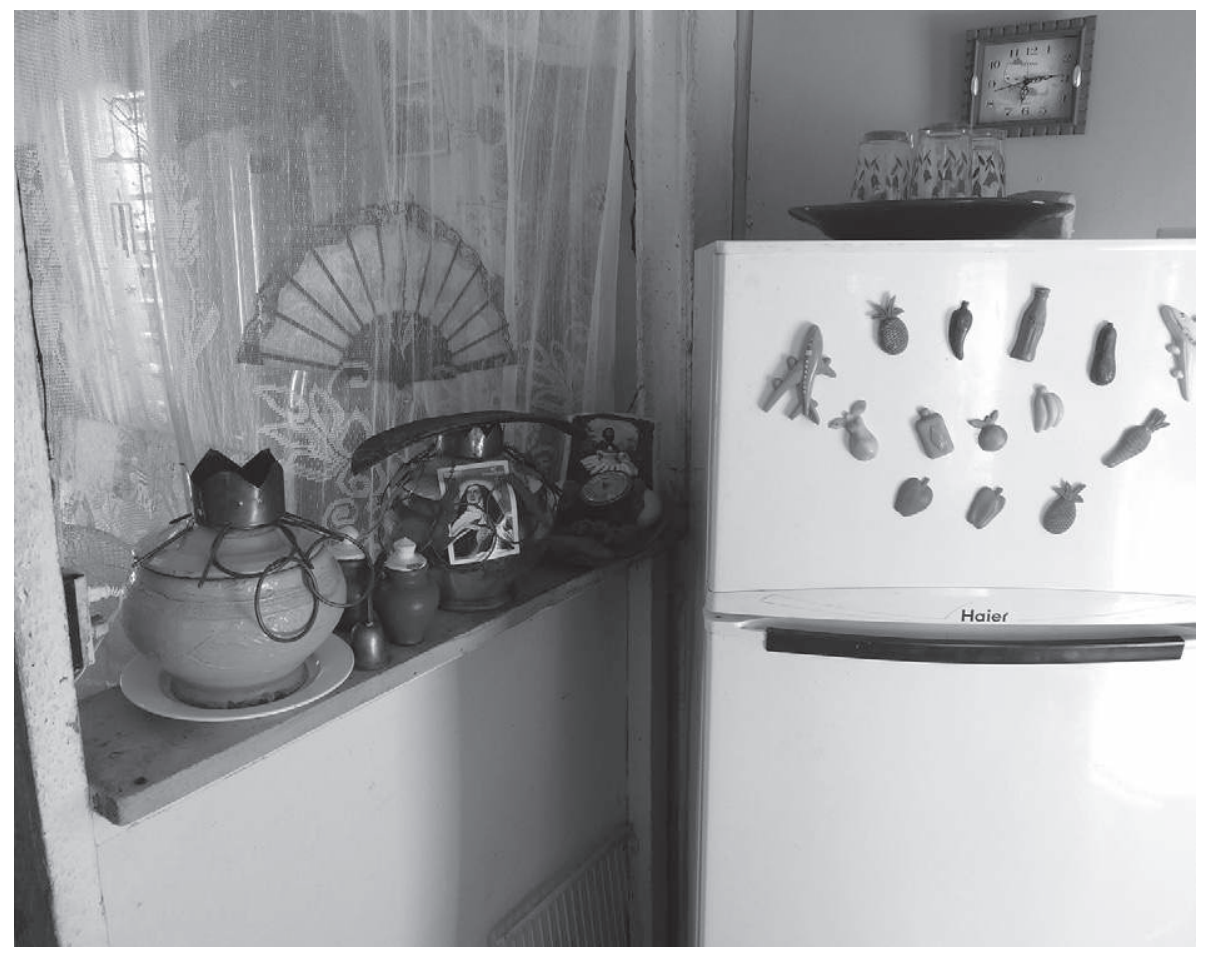

Fig. 14 - Soupières de la santería, installées dans une cuisine.

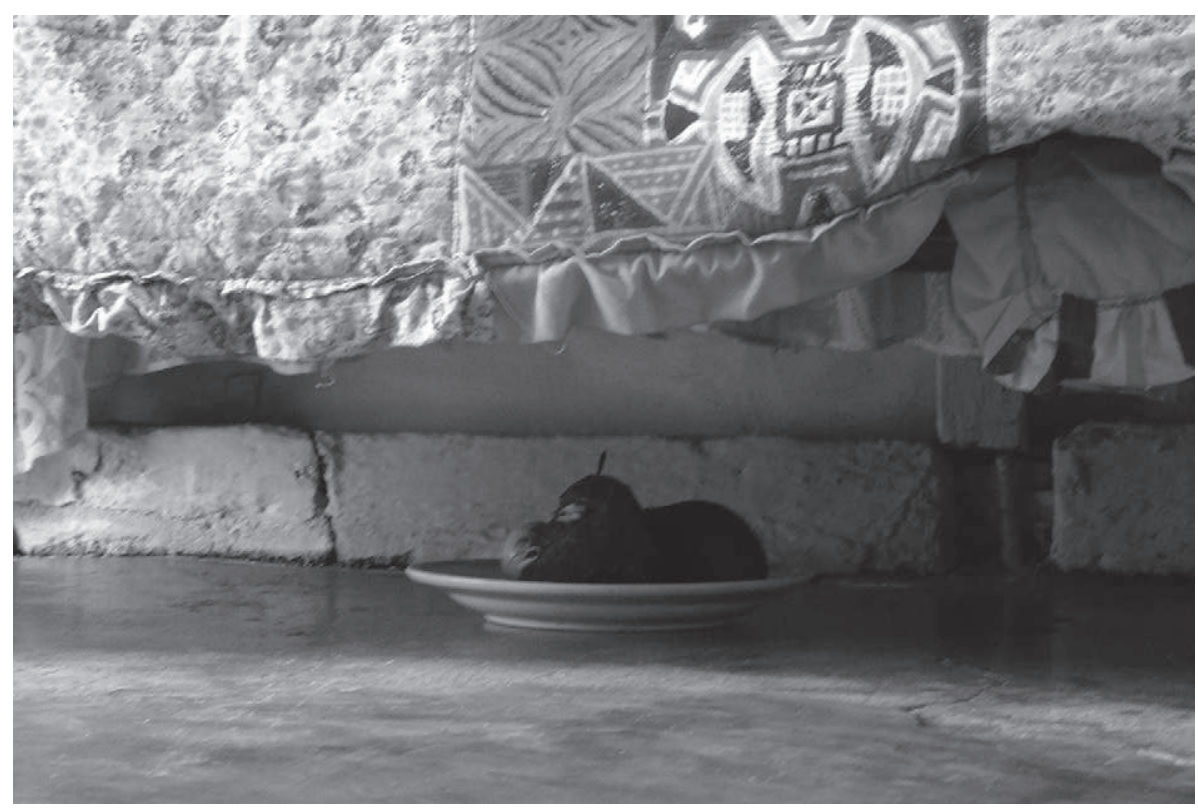

Fig. 15 - Divinité de la santería, cachée sous un canapé. 


\section{Le cas exceptionnel des rituels collectifs}

La ritualité domestique implique aussi l'organisation de cérémonies, annuelles pour la plupart, qui mobilisent l'ensemble de la communauté. À ces occasions, l'équilibre spatial du quotidien se rompt et de nouvelles techniques de compartimentation doivent être mises en place. Certaines pièces subissent ainsi des réaménagements radicaux. Par exemple, une cérémonie du palo monte nécessite parfois qu'on déménage tout le sanctuaire de la petite cour où il est installé, vers le salon, plus vaste et adapté à la réception des invités qui affluent de partout. Or pour opérer ce déplacement, le pratiquant doit se livrer à une série de compromis et de négociations, aussi bien avec les autres membres de la maisonnée qu'avec leurs entités. Il doit mettre un voile sur les effigies catholiques pour ne pas les effrayer, isoler les divinités de la santería par des signes magiques pour éviter qu'elles ne se bagarrent avec les morts du palo, essayer de ne pas heurter la susceptibilité des membres chrétiens, voire les engager dans l'organisation du rituel, etc.

Nous sommes chez le palero Hector, un jour de décembre 2007. Sa mère, qui est santera et spirite, organise une cérémonie de spiritisme syncrétique, autrement appelé « espiritismo cruzado » (spiritisme croisé). La cérémonie se déroule dans le salon, habitat permanent des morts familiaux. On commence par réciter les prières chrétiennes classiques (Notre Père, Ave Maria...) qui ouvrent les cérémonies spirites, puis on entame les oraisons proprement spirites, les plegárias. Ces derniers chants ont pour objectif d'établir le contact avec les esprits et éventuellement de les convoquer sur terre. Trois quarts d'heure après le début de la cérémonie, les morts commencent à se manifester en possédant des médiums. Aussitôt qu'Hector prend conscience de leur arrivée, il se précipite dans la cour de la maison où se trouve son cuarto de fundamento. Il saisit un morceau de craie blanche et trace une grande firma circulaire autour de son autel afin d'isoler les ngangas du reste de la maison (Bonhomme et Kerestetzi 2015). Il faut préciser que le palero avait déjà pris la précaution de soustraire son autel aux regards des visiteurs, en l'entourant d'un grand rideau de plastique opaque. Or la firma qu'il trace maintenant a pour fonction de retenir les nfumbis dans l'espace du cuarto. Selon Hector, sans cette précaution, leur manifestation dans le rituel spirite entraînerait la fuite des " esprits de lumière ", plus faibles, et signerait la fin et l'échec du rituel.

\section{Passerelles esthétiques}

La coexistence religieuse ne repose pas exclusivement sur des processus de mise à distance. Bien au contraire, elle relève aussi, et dans une plus large mesure, de processus de mise en contact et d'interaction ${ }^{5}$. Notamment, par la

5. Pour ce qui concerne d'autres cas similaires d'interactions inter-religieuses entre religions afro-brésiliennes, voir Stefania Capone (1999 et 2016). 
création de passerelles esthétiques : l'affinité morphologique de certains objets avec celle des artefacts des autres cultes présents dans la maisonnée rendant en effet plus fluide le passage d'un univers religieux à l'autre. Par exemple, parmi tous les orichas de la santería, seuls ceux qui sont matérialisés sous la forme de chaudrons en fer ou en terre (les plus sauvages : guerriers, eggun, Ossain) peuvent figurer à proximité des chaudrons des paleros. Doit-on y voir le résultat d'une parenté de nature entre les différentes entités ou d'une parenté esthétique? Ceci vaut aussi pour les symboles chrétiens, spirites et santeros qui partagent le même espace sans qu'il y ait conflit particulier ; leur caractère « domestique ", familial et décoratif jette un pont sur les hiatus idéologiques. Bastide parlait du dialogue des formes symboliques dans les processus syncrétiques (1967). Ici, il est question de dialogue des formes esthétiques qui ouvrent les portes de chaque culte à l'espace de l'autre.

L'exemple de Georgina illustre bien cette notion de passerelle esthétique. Cette femme d'un âge avancé est à la fois palera, spirite, santera et catholique. Elle s'adonne à ces différents cultes de manière particulièrement équitable, bien plus en tout cas que la plupart des Cubains qui cumulent les étiquettes religieuses, privilégiant certains cultes au détriment des autres. La relative égalité que cultive Georgina s'explique en partie par sa manière très organique de briser les frontières religieuses, tant sur le plan rituel qu'esthétique. De plus, elle vit seule dans une petite maison avec cour et peut installer ses artefacts à sa guise sans subir de pression familiale et idéologique, elle a donc tout loisir de laisser libre court à sa créativité syncrétique. Les principales entités qu'elle dispose sont Tomasa, son esprit spirite de prédilection, une Africaine des plantations qui la suit depuis le début de son parcours religieux ; Taita José, Africain également, mais nfumbi du palo monte; et les orichas de la santería et notamment Yemayá qui est son oricha tutélaire.

Nous avons vu que le spiritisme cubain a développé certaines notions qui justifient la libre circulation des esprits d'un culte à l'autre. Au sein des autres cultes s'opèrent également des glissements cosmologiques et conceptuels. Par exemple, la santería établit une parenté entre tous les êtres (vivants ou divins) à travers la notion de parrainage par les divinités. En fait, chaque personne, esprit ou animal (voir chaque végétal et minéral), est considérée comme « fils » ou « fille» d'un oricha. Pour Georgina, cela vaut aussi pour ses esprits protecteurs. Ainsi Tomasa est, comme elle-même, « fille » de Yemayá et Taita José est « fils » de l'oricha Eleguá.

Cette parenté spirituelle entre esprits de différents cultes se traduit chez la vieille pratiquante par une ressemblance esthétique. Georgina vit dans une maison envahie par la présence du religieux. Son salon en particulier est le quartier général de toutes les spiritualités. À l'exception des chaises, on ne voit guère d'objet profane. Ce qui étonne au premier abord, c'est qu'en dépit de la diversité des religions représentées, il se dégage du salon une certaine 


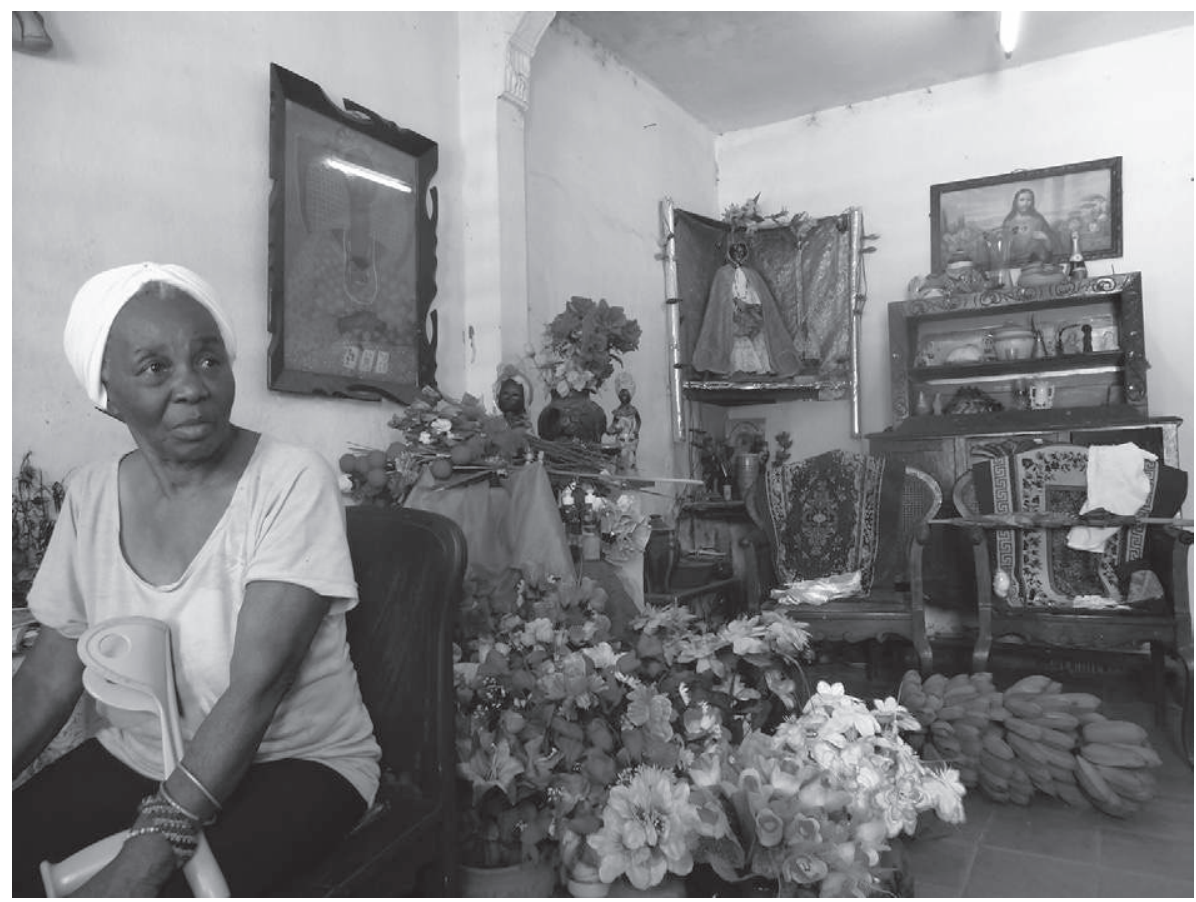

Fig. 16 - Le salon de Georgina.

uniformité stylistique : on croirait être dans un sanctuaire santero (Figure 16). Pour autant, les matérialisations des orichas n'y occupent que le mur arrière et le coin gauche, soit un petit espace. Le reste du salon est un vaste trompel'œil : il ressemble à un espace de la santería alors qu'il est en fait consacré au palo monte et au spiritisme. Il est vrai que les morts spirites n'ont pas de forme particulière, si ce n'est celle des poupées achetées en boutique qu'on peut habiller à sa guise. Si Georgina a choisi d'emprunter le style santero pour ses poupées, c'est parce que, pour elle, les morts spirites sont après tout des « fils » d'orichas. Ainsi, Tomasa, l'Africaine, assise sur une table en hauteur, est représentée par une poupée noire vêtue de bleu comme sa « mère » santera Yemayá. L'Africain Taita José prend la forme d'une poupée en chiffon, noire elle aussi, qui porte un chapeau noir et rouge, les couleurs de son «père » Eleguá. Cette poupée de Taita José est en réalité une doublure de la nganga du palo monte qui se trouve dans un cuarto de fundamento au fond de la cour. Et c'est là qu'on peut identifier de manière limpide la force de l'esthétique : les nfumbis qui prennent la forme d'une nganga ne pourraient jamais occuper le centre d'un salon. Or, déguisés en poupée, ils y trouvent leur pleine place.

En fait, en uniformisant le style des fundamentos de ses esprits et de ses divinités, Georgina a réussi à tous les réunir dans le même espace, tandis que 
d'autres pratiquants qui disposent de possibilités spatiales similaires veillent à bien les séparer physiquement. Dans son salon, il n'y a pas de compartiments. L'uniformité esthétique des objets rituels produit un espace qui exprime la pluralité, tout en restant indivisible. On voit ici comment la notion de passerelle esthétique permet de comprendre non seulement comment la morphologie de l'espace participe à l'atténuation des contradictions idéologiques, mais aussi comment il contribue à faire émerger de nouvelles idées et notions religieuses.

Dans la philosophie religieuse de Georgina, il n'y a pas de limites ontologiques ou idéologiques. Même Jésus est intégré dans son univers rituel avec une fonction et un statut bien particulier, celui de pacificateur; son image dans l'espace de la maison-temple suffit à faire de lui un esprit agissant parmi les autres. Or ce que son cas montre en définitive est que les pratiques interagissent plus facilement quand elles sont médiatisées par des objets qui se ressemblent ou qui s'harmonisent dans l'espace. L'uniformité esthétique des cultes de cette maison, en brisant les frontières spatiales, encadre une sorte de fusion rituelle, ne serait-ce que parce qu'elle permet de réunir dans le même espace indivisible les esprits du palo, du spiritisme, de la santería et le fils du Dieu des chrétiens.

\section{Syncrétisme ad hoc}

On sait que les nfumbis sont insociables - et en ce sens, le palo est la religion afro-cubaine la plus exclusive même si elle n'a aucun mal à intégrer autant d'éléments exogènes qu'elle le juge utile - et qu'ils ne peuvent supporter la présence de divinités ou d'esprits étrangers. Sauf quand ceux-ci sont soumis aux exigences du travail palero. Par exemple, il peut arriver qu'on installe une bóveda spirite à l'entrée d'un cuarto. Ce rapprochement est possible du fait de l'affinité ontologique des morts du spiritisme avec les nfumbis, esprits des morts eux aussi. Mais surtout parce que les morts spirites sont jugés trop désincarnés ou trop faibles pour menacer l'action des nfumbis. Les anges gardiens spirites sont ainsi relégués au statut d'assistants venus seconder le « travail » palero en prodiguant leurs bonnes grâces. Évidemment, leur espace est bien délimité dans la pièce du palo monte : il est souvent séparé à l'aide des murs et de signes magiques (Figure 17, page suivante). Il faut noter que ce processus d'appropriation symbolique ne fonctionne pas dans les deux sens. D'un point de vue spirite, la compagnie et le voisinage des nfumbis « maléfiques » apparaissent comme des menaces pour leurs morts « épurés » et bienveillants, on veillera donc à soigneusement les maintenir séparés. Dans une même logique, lorsqu' on voit dans certains sanctuaires paleros dits « chrétiens » des croix et des crucifix, il ne faut pas en déduire que le rapport entre ces deux univers symboliques est équilibré, loin de là : les symboles chrétiens ont en quelque sorte été digérés, « réinterprétés 》 (Herskovits 1941 ; Bastide 1967), par l'univers palero. Selon Bastide, cette réinterprétation implique tant un travail sur le sens que sur la forme de l'objet approprié : en effet les crucifix sont couverts de firmas et sont 


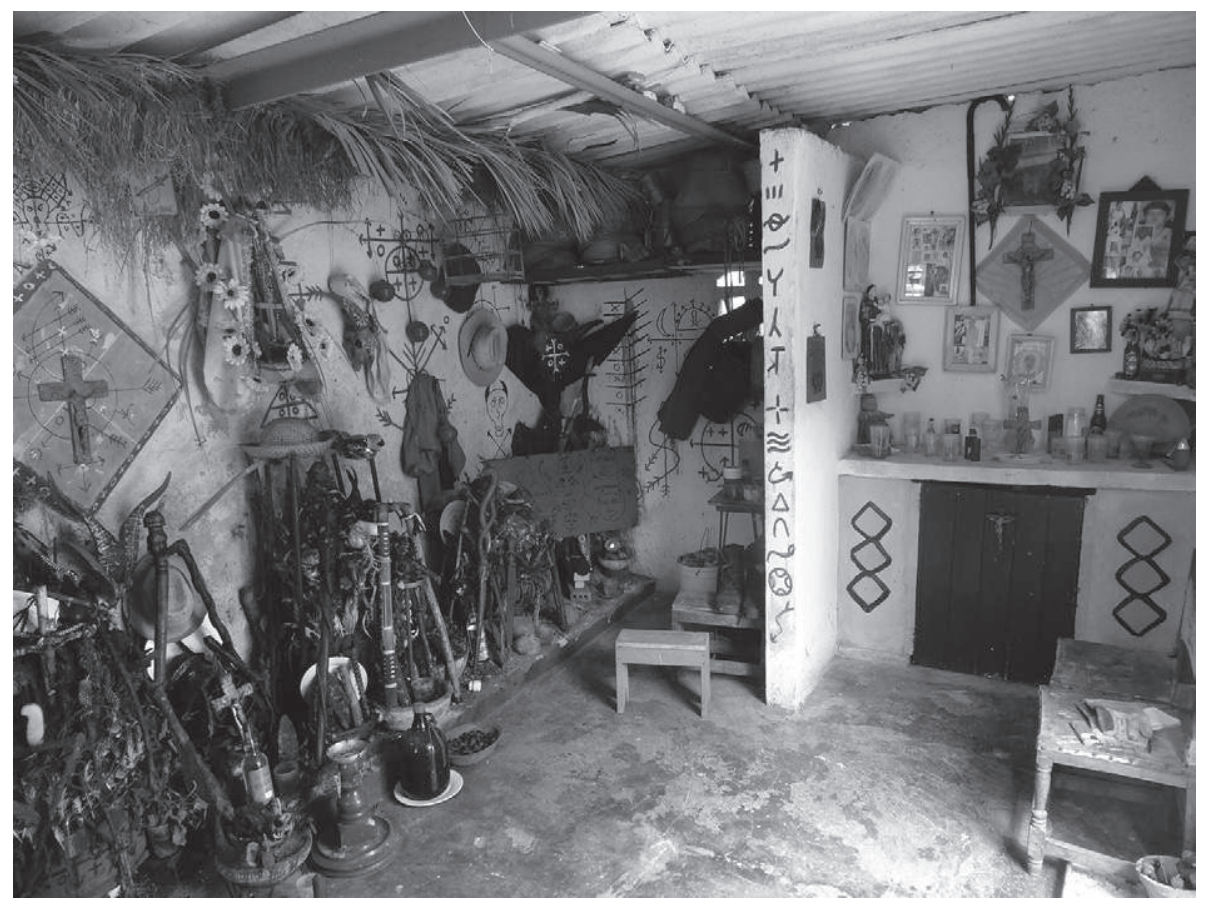

Fig. 17 - Autel spirite (à droite) dans un cuarto de fundamento.

Un mur et des firmas le séparent des ngangas du palo monte.

remplis de substances magiques, ils sont régulièrement aspergés du sang des sacrifices, activités assez peu chrétiennes somme toute. Si dans ces situations l'argument théologique semble primer, il faut noter que l'esthétique a aussi un rôle à y jouer : l'aspect et l'odeur de putréfaction des objets du palo monte sont très difficilement adaptables et combinables, tandis que les symboles spirites et chrétiens sont assez discrets et s'intègrent mieux dans des univers hétérogènes.

L'anthropologie s'est souvent intéressée au fait que différentes religions pouvaient se combiner sans susciter de troubles cognitifs auprès de leurs fidèles, malgré les évidentes contradictions que leur combinaison générait. Ceci a donné lieu à de passionnants travaux qui ont éclairé les logiques et les procédés qui sont en jeu au cœur du syncrétisme : les modalités d'équivalences entre divinités hétérogènes, le syncrétisme du masque, l'acculturation formelle, le principe de réinterprétation, le bricolage... (Lévi-Strauss 1962 ; Bastide 1967 ; Mary 1999). Si dans l'exemple précédent, il est question d'appropriation instrumentale, c'est parce que certains processus du syncrétisme afro-cubain s'opèrent également selon la logique du besoin pragmatique ; il s'agit en somme d'un syncrétisme ad hoc qui puise dans toutes les affordances matérielles de son environnement, y compris spatiales. Nous l'avons vu, les raisons pratiques, les besoins du 
moment et les volontés particulières contraignent les modalités syncrétiques au point d'en éclipser parfois les divergences inter-religieuses. Et si certains pourraient voir ici des processus individualisant dépourvus de valeurs et de principes collectifs, il ne s'agit pour autant pas de ce qu'André Mary (1999, p. 196) qualifie de religiosité « à la carte ». Il faut plutôt y voir une prolongation d'un mode de vie dans le domaine religieux. Les Cubains disent qu'il faut « inventer » (inventar) le quotidien, c'est-à-dire être sans cesse en alerte pour trouver des solutions ingénieuses à la privation et à tous les besoins matériels qui peuvent se présenter à tout moment. C'est cette posture débrouillarde qui s'exprime dans leur manière de faire coexister leurs pratiques religieuses, au point d'en devenir presque un principe matriciel.

\section{Négociations, compromis spatiaux}

Si nous avons jusqu'ici présenté certains principes généraux structurant les écosystèmes domestiques, nous allons maintenant nous intéresser davantage aux dynamiques relationnelles qui accompagnent les processus de partage de l'espace domestique entre plusieurs religions. Nous verrons que la distribution spatiale spécifique des objets rituels est aussi une affaire de politique domestique.

\section{Lieux d'action et d'inaction : famille rituelle, ritualité familiale}

Les exemples suivants vont nous permettre de nous rendre compte de l'impact de l'emplacement des objets sur la pratique religieuse. Nous verrons que certains lieux de la maison participent à l'intensification de la ritualité. Ces « centres spatiaux énergétiques » augmentent la fréquence rituelle au sein de l'habitat, soit parce qu'ils sont situés dans des lieux stratégiques, soit parce que leur agencement permet de déployer un répertoire gestuel plus étendu. Cette centralité les intègre pleinement dans le quotidien de la famille : les adeptes leur offrent des plats cuisinés, des fleurs, leur posent des questions, dînent en leur présence, invitent les voisins à s'entretenir avec eux... Cette situation fait que la ritualité de ces « centres » (géographiques, sociaux) ne peut être pensée indépendamment de la famille et de ses routines. Placer les objets rituels aux endroits les plus fréquentés de l'habitat revient à multiplier les occasions d'interaction entre les divinités et les occupants de la maison. Leur coprésence incitant à l'action, on peut parler ici d'une ritualité induite ou opportuniste - au sens d'opportunité.

Au contraire, certains emplacements « périphériques » ou à faible teneur interactionnelle ne prédisposent pas à la pratique rituelle et conduisent à l'inactivité. Disniavi, un pratiquant du palo monte de Cienfuegos, a dû ardemment batailler avec son épouse catholique pour pouvoir installer son chaudron palero dans leur modeste appartement. Le malheureux ne s'est vu allouer pour tout espace religieux que le placard situé sous l'évier de la cuisine. Pour lui, la pratique du 
palo monte à la maison est ainsi réduite à portion congrue car il doit composer de surcroît avec la présence d'une famille nombreuse. Il ne peut ainsi jouir ni de l'intimité avec son mort, ni de la possibilité de recevoir des clients. C'est donc chez son initiateur et voisin qu'il passe aujourd'hui le plus clair de son temps libre. D'ailleurs, il y participe davantage aux routines familiales qu'à celles de sa propre maison.

Et cela n'est pas anecdotique. La distance de la famille à une pratique peut influencer jusqu'à son déroulement et sa ritualité. Évoquons ici le cas de deux paleros : José et Pancho. L'un dispose d'une arrière-cour, l'autre non. Le premier a bâti au fond de sa cour une cabane en bois, dans laquelle il a installé sa nganga; le second n'a eu d'autre choix que d'installer son chaudron dans une pièce de la maison. Dans le cas de José, le sanctuaire est exclusivement réservé à la pratique du palo et favorise le développement d'une pratique solitaire. Dans le cas de Pancho, la famille est présente, voire impliquée, dans la pratique du palo monte : le fils est chargé de trouver les animaux à sacrifier, la sœur a pour mission de fabriquer les amulettes... Et lorsqu'un client vient consulter ce palero, il échange quelques paroles de courtoisie avec sa mère et se voit offrir un café par sa belle-fille. Ce n'est donc pas un hasard si les deux paleros ont développé des pratiques vraiment différentes. Celui qui a séparé religion et famille cultive l'image d'un sorcier ténébreux et se déclare prêt à accomplir tout type de magie, qu'elle soit nocive ou salvatrice. À l'inverse, celui qui fait fusionner les sphères familiale et religieuse déclare avec emphase travailler exclusivement au « service du bien » et comme pour signifier encore mieux son altruisme, il a installé une grande image du Christ dans le salon où attendent ses clients. L'espace occupé par les différents cultes au sein de l'habitat, ainsi que leur distance relative à la vie sociale d'une famille sont révélateurs de l'identité rituelle des pratiquants et de l'image publique de leur culte. La santería, mise en valeur dans les pièces publiques les plus fréquentées, cultive spatialement sa bonne réputation alors que le palo, d'ordinaire pratiqué à la périphérie de la maison, par exemple dans la confidentialité d'une cabane modeste, nourrit volontiers les fantasmes sorcellaires tandis qu'il entretient la mauvaise réputation de ses adeptes.

\section{Religions exclusives : discrétion, neutralité, échange}

Nous nous intéresserons maintenant à la manière dont les pratiquants des cultes afro-cubains et ceux de certaines confessions chrétiennes « exclusives » parviennent à vivre ensemble. À Cuba, malgré la forte présence des témoins de Jéhovah, des pentecôtistes et plus généralement des évangéliques, tous idéologiquement très hostiles à d'autres formes religieuses, on peut encore parler d'une coexistence religieuse relativement paisible. Les exemples qui vont suivre montrent comment cette coexistence s'exprime au quotidien dans les espaces domestiques plurireligieux. Évidemment, ces maisons sont, par 
définition et par nécessité, des lieux de tolérance, au point qu'il suffit parfois de quelques arrangements spatiaux pour accommoder les idées de chacun. Et si les exemples présentés ici relèvent pour une majorité d'entre eux de l'exception, ils rendent compte avec force réalisme de plusieurs habitats qu'il nous a été donné d'observer dans la province de Cienfuegos.

Dans la maison d'Alicia, assez spacieuse, vivent trois personnes : elle, sa fille, et sa grand-mère de 98 ans. Elle et sa fille sont santeras et sa grand-mère est témoin de Jéhovah, depuis plus de 20 ans (elle était baptiste auparavant). Pour cette dernière, « la santería est chose du Diable ». Pourtant, grâce à certains réaménagements discrets, elle parvient à vivre avec sa famille santera sans trop de tensions. Il faut noter que les artefacts santeros sont ici trop nombreux pour être dissimulés dans un placard. Pour respecter la grand-mère, on est obligé de les cacher derrière le réfrigérateur, dans un coin de la chambre, à côté d'un placard, au-dessus d'une armoire (Figure 18). On doit également dissimuler l'activité rituelle, en couvrant par exemple les offrandes aux divinités d'un tissu opaque, ou bien en optant pour la discrétion : si l'on doit organiser un rite collectif, on doit déplacer tous les objets cultuels dans la cour de la maison et cantonner le rite à cet espace. Bien sûr, on doit aussi veiller à ce que la grand-mère reste à

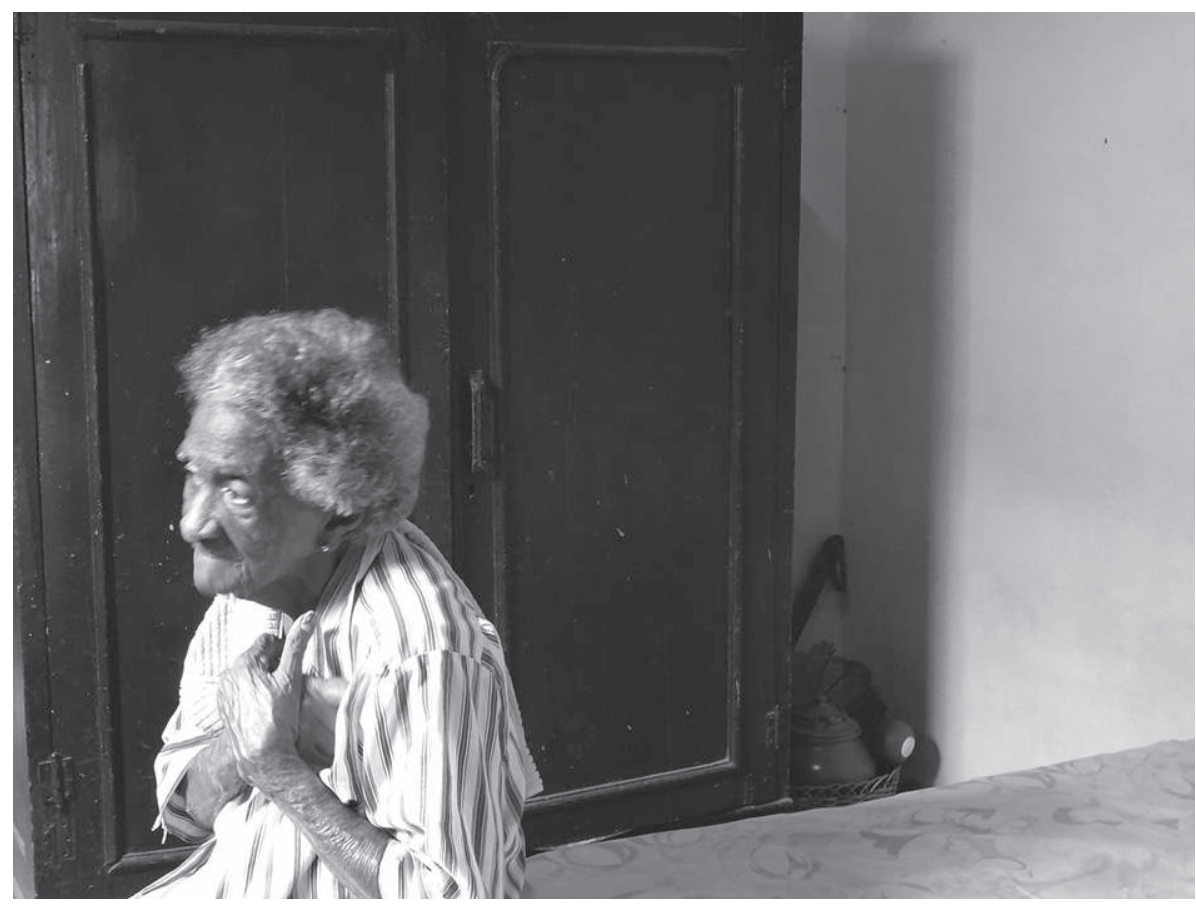

Fig. 18 - La grand-mère témoin de Jehova et une soupière de la santería, cachée à côté du placard. 
l'autre extrémité de la maison. Il subsiste néanmoins un problème, car pendant les rituels afro-cubains, toutes les personnes qui vivent dans la maison doivent être purifiées rituellement afin que les mauvaises influences soient définitivement expulsées de l'habitat, sans exception aucune. Or il n'est pas question que la grand-mère accepte. Heureusement, Iscanday, le fils d'Alicia, lui-même santero, a trouvé une solution. Il cache un pigeon purificatoire derrière son dos et, sans que la grand-mère s'en rende compte, il le passe trois fois au-dessus de sa tête pour la « nettoyer » puis le relâche par la porte de l'entrée afin que le mal s'évapore aux quatre vents. Il faut noter que la grand-mère n'est pas aussi intolérante que la plupart de ses coreligionnaires. Comme le note son arrièrepetit-fils : «Elle respecte. Quand on organise une fête de la santería, à la fin, elle vient boire et papoter avec nous, comme si c'était un événement culturel. Et quand on met un verre d'eau à quelqu'un [l'avatar d'une divinité], elle ne le jette pas, ni rien. La chose qu'elle aime le moins est quand vient quelqu'un de sa religion et qu'il y a par exemple un récipient avec des plumes à la vue de tous ». C'est la discrétion donc ici qui rend possible la cohabitation religieuse. Le terme n'est pas choisi au hasard. La discrétion est de manière générale une attitude au profit de la bonne sociabilité. Dans certaines maisons pluriconfessionnelles, elle s'érige en véritable valeur familiale, un comportement « comme il faut», une politesse. «Elle (ou il) respecte (ma religion) » est aussi le politiquement correct pour beaucoup de Cubains - il ne faut pas oublier que la pluralité (religieuse, ethnique, sociale) a une longue histoire à Cuba.

Que se passe-t-il quand les membres d'un couple sont affiliés à des religions antagoniques et qu'ils partagent de surcroît un espace restreint ? C'est cette épineuse équation que doivent résoudre au quotidien Yaima, évangéliste, et Alejandro, palero. Difficile en effet de trouver deux religions plus incompatibles : pour les évangélistes, les paleros sont presque une manifestation du Diable. Disons-le d'emblée, Yaima a l'esprit ouvert et le fait que sa propre mère soit palera et spirite n'y est pas étranger. Pourtant, il a tout de même fallu instaurer des règles pour permettre ce modus vivendi, au premier rang desquelles figure l'obligation pour les deux parties de maintenir la neutralité religieuse dans leur toute petite maison. Alejandro possède une cabane en bois au fond de la cour où il héberge ses chaudrons rituels et il n'y effectue ses rituels que lorsque sa femme est absente. Yaima, quant à elle, ne pratique qu'à l'extérieur du foyer. Elle se rend à l'Église ou dans les maisons de ses coreligionnaires pour prier. Elle respecte à la lettre le pacte scellé avec son compagnon, puisque les murs de sa maison sont vierges du moindre crucifix ou du plus petit extrait de l'Évangile que ses coreligionnaires ont pour habitude d'accrocher chez eux.

Les marques de respect entre membres de religions antagoniques sont monnaie courante dans les espaces plurireligieux cubains. Parfois, il s'agit simplement d'éviter le sujet et de fermer de manière bienveillante les yeux sur les pratiques de l'autre, parfois cela peut aller beaucoup plus loin jusqu'à l'exécution de gestes 
rituels en direction du culte « adverse ». L'anecdote racontée par le palero Elier fournit une assez bonne illustration de cette sorte d'osmose interreligieuse. Le cuarto de fundamento de ce palero est installé dans une petite construction en ciment, sise au pied du petit immeuble où il réside. Sa tante, elle aussi résidente de l'immeuble, possède l'appartement mitoyen du cuarto d'Elier ; les deux sont au rez-de-chaussée et partagent une cour. Fervente chrétienne épiscopale, la tante a organisé chez elle lors de la semaine de Pâques une fête religieuse où elle a invité son pasteur, ses coreligionnaires mais aussi son neveu Elier. Un peu plus tard dans la soirée, au moment de servir le repas aux invités, la tante d'Elier se rend dans le cuarto de son neveu afin de servir la première assiette aux ngangas. Pour ne « pas les vexer », dit-elle mais aussi, sans doute, pour gagner leurs faveurs. Elier respecte aussi la religion de sa tante. Comme nombre de paleros et de santeros, pour cette semaine de Pâques, il a couvert ses artefacts d'un tissu blanc, signe de leur inactivité et donc de son respect pour le Christ qui désapprouve le «travail » afro-cubain. Le fait de posséder un cuarto offert au regard de ses voisins chrétiens n'y est sans doute pas étranger. Sans cela, il n'est pas dit qu'Elier se serait abstenu de palo toute une semaine durant. En effet, certains paleros considèrent que la semaine de Pâques est une période très propice à l'exercice de la sorcellerie, quand « Jésus est mort ». Pour Elier, l'effort de neutralisation rituelle de ses ngangas peut aussi bien être vu comme une marque d'affection pour sa tante que comme une promesse de gain de respectabilité auprès du voisinage. Ici, une affaire relationnelle s'est muée en composante rituelle.

\section{Le cas des " professionnels 》 de la religion : synergie, interaction}

Certains initiés ont fait de leur pratique un métier, parfois à temps plein. Leur présence au sein d'une maison de spiritualité mixte peut profondément influencer la morphologie spatiale. Si par exemple dans la maison, il y a un pratiquant dont les consultations constituent la source principale des revenus familiaux, c'est toute la famille, indépendamment des appartenances religieuses de chacun, qui pourra se retrouver au service d'une religion unique. Dans ce type de maison, la ritualité est la routine familiale : les gestes rituels, aussi spectaculaires soient-ils (sacrifices, production de gerbes de feu), seront pour chacun des habitants d'une banalité quotidienne.

Quand deux professionnels habitent ensemble, il arrive parfois que des collaborations inter-religieuses se mettent en place. Je reprendrai l'exemple du palero Hector qui vit avec sa mère Juana, santera et spirite. Quand Hector reçoit en consultation, parfois, son oracle révèle que le problème de son client a de meilleures chances d'être résolu par la santería. Sa mère peut donc prendre le relais, ce qui est bien commode. Une philosophie complémentariste justifie ces glissements : «Le savoir a été réparti ; la sagesse a été répartie. Le palo a sa 
manière de travailler, la santería a sa manière de travailler... Je peux être un très bon palero mais d'autres peuvent être de très bons spirites », explique Hector. Cette répartition du savoir religieux se traduit par une forme spatiale dans leur maison. Comme celle-ci est assez vaste, elle offre la possibilité à chacun des deux pratiquants de répartir leurs divinités respectives de façon à ce qu'elles s'épanouissent dans des environnements adaptés (le domaine d'Hector et du palo monte est la cour, celui de Juana est le salon; entre eux, il y a la cuisine et les chambres pour les séparer franchement), mais aussi pour créer pour chacun un « cabinet de consultation » à la hauteur de leurs attentes.

Si le facteur financier semble œuvrer ici pour la pacification des rapports entre les différentes religions au sein de l'habitat, il n'en constitue pas l'unique raison. Comme cela arrive parfois, les consultations domestiques peuvent fournir à chacun un rôle, un objectif, une place et des gains (spirituels, économiques, éducatifs), peu importe son appartenance. La pratique religieuse professionnelle devient ainsi un mode de vie pour la famille qui, organisant l'activité de ses membres autour d'un but commun, peut lui apporter cohésion et équilibre.

\section{Conclusion}

Une approche anthropologique classique de la pluralité religieuse qui serait centrée sur les principes théologiques, sur les mythes, sur les structures rituelles ou même sur les dynamiques sociologiques la traversant, aurait peut-être du mal à rendre compte du caractère éminemment pragmatique et situationnel de la coexistence de différents cultes mis à proximité. Cet article a considéré l'espace domestique, et l'espace généralement, non pas comme un simple contenant de la pluralité religieuse, un cadre ou une toile de fond pour ce qui se déroule en son sein, mais comme un véritable facteur, voire un moteur, des modalités de coexistence religieuse.

Nous nous sommes notamment intéressés à la question de l'esthétique de l'espace domestico-rituel. L'esthétique ici n'a pas été considérée comme un simple produit des idéologies, des traditions et des pratiques mais plutôt comme ce qui produit des idéologies et des pratiques ; elle est dynamique, édifiante, et ce, de manière tout à fait pragmatique. Nous avons vu que la place qu'occupent les objets dans l'espace domestique relève pour une large part de leur capacité à se combiner et à se confondre avec le mobilier et la décoration d'une maisonnée. Et ceci n'est pas anecdotique. En effet, les affordances de l'espace ont des conséquences liturgiques : elles peuvent soit inciter à la ritualité (ce qu'on a appelé une « ritualité induite, opportuniste ») et augmenter sa fréquence, soit au contraire la restreindre. Bien sûr, la place qu'occupent les objets dans l'espace dépend de plusieurs paramètres et leur étude ne peut que concerner les approches situationnelles. Or dans le cadre des religions aussi plurielles et idiosyncrasiques que les cultes afro-cubains, c'est justement en identifiant ces variables qu'on peut en dégager les structures. 
La notion de passerelle esthétique nous a permis de montrer comment l'affinité morphologique de certains espaces ou de certains objets permettait de jeter un pont sur les hiatus idéologiques. Ce dialogue des formes esthétiques a la vertu de réconcilier des religions conceptuellement éloignées, en opérant des rapprochements sur le contenant, plutôt que sur le contenu. Cette réflexion sur la question des passerelles, qui insiste tout particulièrement sur les connexions rituelles, nous amène inévitablement à poser la question de la coupure et du cloisonnement des cultes et des rites. En effet, de même qu'il existe des ponts esthétiques, il existe aussi des délimitations qui sont censées garder à distance ce qui doit l'être et qui sont tout aussi productives. C'est par ces passerelles et par ces frontières qu'on arrive souvent dans une maison cubaine à des compromis religieux qui peuvent prendre, du coup, soit la forme d'interaction rituelle soit de tolérance et de respect.

Nous sommes ainsi arrivés à la notion de syncrétisme ad hoc. Il s'agit d'un syncrétisme qui se produit par la synergie de plusieurs facteurs qu'on peine habituellement à mettre sur un pied d'égalité. Or, qu'il s'agisse de processus syncrétiques ou plus généralement de modalités de coexistence de plusieurs religions dans l'espace confiné des maisons afro-cubaines, nous avons vu que les contraintes économiques, spatiales, relationnelles, représentationnelles et cosmologiques s'entrelacent pour former un système et édifier la morphologie rituelle d'une maison-temple. Ici, les concepts se traduisent en matière (la parenté symbolique entre divinités étrangères se traduit en parenté esthétique dans la forme de leurs avatars) ; les relations de parenté deviennent des composantes cosmologiques ; les exigences rituelles se transforment en routines familiales. On arrive ainsi au constat qu'on ne peut séparer religion, rapports familiaux et spatialité. Dans l'espace confiné d'une maison, ce type de catégorie conceptuelle perd complètement son sens habituel : la famille devient une famille ritualisée ; la religion devient une ritualité familiale.

La problématique spatiale dépasse largement le champ d'étude des religions afro-cubaines. Le caractère concret et empirique de la méthodologie proposée en fait un modèle potentiellement transposable aux situations qui nécessitent d'examiner les modalités de coexistence de plusieurs religions dans un espace donné. Si ce travail, centré sur l'espace et la matérialité, produit des résultats pertinents dans le cas du complexe religieux afro-cubain du fait de l'usage immodéré qui y est fait des objets et des substances, il a aussi la vertu de nous rendre attentifs à la manière dont on transforme un espace a priori non spécialisé en environnement propice à la pratique d'une religion donnée. De manière générale, cette étude socio-anthropologique des matérialités et des spatialités religieuses afro-cubaines permet de comprendre comment on fabrique du religieux et comment on vit avec l'altérité dans les faits. *

\footnotetext{
* Manuscrit reçu en juillet 2017, accepté pour publication en février 2018.
} 


\section{Références bibliographiques}

ANDRÉ Jacques

1987 L'inceste focal : dans la famille noire antillaise. Crimes, conflits, structure, Presses universitaires de France, Paris.

ArgYriadis Kali

1999 La Religion à La Havane, Archives contemporaines, Paris.

BASTIDE Roger

1967 Les Amériques noires, L'Harmattan, Paris.

Bonhomme Julien et Katerina KerestetzI

2015 « Les signatures des dieux. Graphisme et action rituelle dans les religions afro-cubaines », Gradhiva, 22, p. 74-105.

Brown David H.

2003 Santeria Enthrone: art, ritual, and innovation in an Afro-Cuban religion, University of Chicago Press, Chicago, London.

CABrera Lydia

1986 La regla Kimbisa del Santo Cristo del buen viaje, Universal, Miami-Florida.

CAPONE Stefania

1999 La quête de l'Afrique dans le Candomblé : pouvoir et tradition au Brésil, Karthala, Paris.

2016 «The Pai-de-santo and the Babaláwo. Religious interaction and ritual rearrangements within Orisha religion ", in Jacob K. Olupona et Rebecca Abiodun Oyedeji (dir.), Ifá divination, knowledge, power, and performance, Indiana University Press, Bloomington/Indianapolis, p. 213-223.

Dianteill Erwan

2000 Des dieux et des signes : initiation, écriture et divination dans les religions afro-cubaines, Éditions de l'École des hautes études en sciences sociales, Paris.

EsPírito SANTo Diana

2009 Developing the dead: the nature of knowledge, mediumship, and self in Cuban espiritismo, thèse de doctorat en anthropologie, University College of London, London.

2015 Developing the dead mediumship and selfhood in Cuban espiritismo, University Press of Florida, Gainesville.

Flores-Peña Ysamur et Roberta J. Evanchuk

1994 Santería garments and altars: speaking without a voice, University Press of Mississippi, Jackson.

Gell Alfred

1998 Art and agency: an anthropological theory, Oxford University Press, New York, Oxford. 
GiBson James J.

1977 «The theory of affordances », in Robert Shaw et John Bransford (dir.), Perceiving, acting, and knowing, toward an ecological psychology, Halsted Press Division-Wiley, New York.

Giobellina Brumana Fernando

1994 Las formas de los dioses: categorías y clasificaciones en el Candomblé, Universidad de Cádiz, Cádiz.

Giobellina Brumana Fernando et Elda Gonzales Martínez

1989 Spirits from the margin. Umbanda in Sao Paolo: a study in popular religion and social experience, Acta Universitatis Upsaliensis, Uppsala.

GoBIn Emma

2012 Un complexe sacerdotal cubain: les santeros, les babalaos et la réflexivité critique, thèse de doctorat en ethnologie, Université Paris Ouest Nanterre La Défense, Nanterre.

GonzÁlez Huguet Lydia

1968 «La casa templo en la Regla de Ocha », Etnología y Folklore, 3, p. 31-64.

HANKS William

1996 «Exorcism and the description of participant roles », in Michael Silverstein et Greg Urban (dir.), Natural Histories of Discourse, The University of Chicago Press, Chicago, p. 160-202.

Herskovits Melville J.

1941 The Myth of the negro past, Harper \& Brothers Publishers, New York/London.

HolbraAd Martin

2007 « The power of powder: multiplicity and motion in the divinatory cosmology of Cuban Ifá (or mana, again) », in Amiria Henare, Martin Holbraad et Sari Wastell (dir.), Thinking through things: theorising artefacts ethnographically, Routledge, London/New York, p. 189-225.

Johnson Paul Christopher

2002 Secrets, Gossip, and Gods: the transformation of Brazilian Candomblé, Oxford University Press, Oxford.

KERESTETZI Katerina

2016 Vivre avec les morts à Cuba : réinvention et transmission religieuse dans le palo monte afro-cubain, Karthala (Religions contemporaines), Paris.

LÉvi-STRAuss Claude

1962 La pensée sauvage, Plon, Paris.

LÓPEZ VALDÉs Rafael Leo

1985 Componentes africanos en el etnos cubano, Ciencias, La Havana.

MARY André

1999 Le défi du syncrétisme. Le travail symbolique de la religion d'Éboga (Gabon), Éditions de l'École des hautes études en sciences sociales, Paris. 
Katerina KeRESTETZI

MARY André

2000 Le bricolage africain des héros chrétiens, Le Cerf, Paris.

Mason Michael Atwood

1992 Living Santería. Rituals and experiences in an Afro-Cuban religion, Smithsonian Institution Press, Washington/London.

ORTIz Fernando

1986 [1926] Los Negros Curros, Ciencias Sociales, La Havana.

PALMié Stephan

2002 Wizards \& scientists: explorations in Afro-Cuban modernity \& tradition, Duke University Press, Durham/London.

STOLCKe Verena

1992 «The family in Jamaica, Cuba, and Brazil », in Elsa Berquó et Peter Xenos (dir.), Family systems and cultural change, Clarendon Press, Oxford, p. 125-143. 